\title{
Physical properties of AM CVn stars: New insights from Gaia DR2 ${ }^{\star}$
}

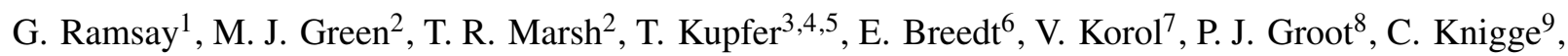 \\ G. Nelemans ${ }^{8,10}$, D. Steeghs ${ }^{2}$, P. Woudt ${ }^{11}$, and A. Aungwerojwit ${ }^{12}$ \\ 1 Armagh Observatory and Planetarium, College Hill, Armagh BT61 9DG, UK \\ e-mail: gavin.ramsay@armagh.ac.uk \\ 2 Department of Physics, University of Warwick, Coventry CV4 7AL, UK \\ 3 Kavli Institute for Theoretical Physics, University of California, Santa Barbara, CA 93106, USA \\ ${ }^{4}$ Department of Physics, University of California, Santa Barbara, CA 93106, USA \\ 5 Division of Physics, Mathematics and Astronomy, California Institute of Technology, Pasadena, CA 91125, USA \\ ${ }^{6}$ Institute of Astronomy, University of Cambridge, Madingley Road, Cambridge CB3 OHA, UK \\ ${ }^{7}$ Leiden Observatory, Leiden University, PO Box 95132300 RA, Leiden, The Netherlands \\ 8 Department of Astrophysics/IMAPP, Radboud University, PO Box 9010, 6500 GL Nijmegen, The Netherlands \\ 9 Department of Physics and Astronomy, University of Southampton, Southampton SO17 1BJ, UK \\ ${ }^{10}$ Institute of Astronomy, KU Leuven, Celestijnenlaan 200D, 3001 Leuven, Belgium \\ 11 Inter-University Institute for Data Intensive Astronomy, Department of Astronomy, University of Cape Town, Private Bag X3 \\ Rondebosch 7701, South Africa \\ 12 Department of Physics, Faculty of Science, Naresuan University, Phitsanulok 65000, Thailand
}

Received 17 September 2018 / Accepted 14 October 2018

\begin{abstract}
AM CVn binaries are hydrogen deficient compact binaries with an orbital period in the 5-65 min range and are predicted to be strong sources of persistent gravitational wave radiation. Using Gaia Data Release 2, we present the parallaxes and proper motions of 41 out of the 56 known systems. Compared to the parallax determined using the HST Fine Guidance Sensor we find that the archetype star, AM CVn, is significantly closer than previously thought. This resolves the high luminosity and mass accretion rate which models had difficulty in explaining. Using Pan-STARRS1 data we determine the absolute magnitude of the AM CVn stars. There is some evidence that donor stars have a higher mass and radius than expected for white dwarfs or that the donors are not white dwarfs. Using the distances to the known AM CVn stars we find strong evidence that a large population of AM CVn stars has yet to be discovered. As this value sets the background to the gravitational wave signal of LISA this is of wide interest. We determine the mass transfer rate for $15 \mathrm{AM} \mathrm{CVn} \mathrm{stars} \mathrm{and} \mathrm{find} \mathrm{that} \mathrm{the} \mathrm{majority} \mathrm{has} \mathrm{a} \mathrm{rate} \mathrm{significantly} \mathrm{greater} \mathrm{than} \mathrm{expected} \mathrm{from} \mathrm{standard} \mathrm{models.} \mathrm{This} \mathrm{is} \mathrm{further}$ evidence that the donor star has a greater size than expected.
\end{abstract}

Key words. accretion, accretion disks - stars: distances - white dwarfs

\section{Introduction}

AM CVn stars occupy the extreme short period tail of stellar binaries, with observed orbital periods in the range $\sim 5-65 \mathrm{~min}$. They consist of white dwarfs accreting material from Roche lobefilling companion stars, typically a lower mass white dwarf or a semi-degenerate helium-rich star. The mass transfer in these binaries is driven by gravitational wave radiation and they are expected to be strong sources of low frequency gravitational waves (Nelemans et al. 2004). In particular, the fact that their binary properties such as orbital period and mass ratio can be measured from electromagnetic observations makes them useful as "verification sources" for the Laser Interferometer Space Antenna (LISA) mission. In a separate paper, we predict the gravitational wave strain of the 16 currently-known LISA verification binaries, 11 of which are AM CVn stars (Kupfer et al. 2018).

There are three proposed channels for the formation of AM CVn stars, but their relative importance is not yet clear.

* Photometry of AM CVn stars is also available at the CDS via anonymous ftp to cdsarc.u-strasbg.fr (130.79.128.5) or via http://cdsarc.u-strasbg.fr/viz-bin/qcat?J/A+A/620/ A141
The population synthesis models of Nelemans et al. (2001a) suggest that the majority of AM CVn stars form from double white dwarf binaries that evolve closer together as a result of gravitational wave radiation, and start mass transfer at orbital periods of 2-3 min (Paczyński 1967). Given these small orbital separations, the initial mass transfer occurs as direct impact, which will lead to unstable mass transfer and hence merger in the majority of cases. The fraction of double white dwarfs which survive to become stable mass transferring AM CVn stars is highly uncertain, and depends on the efficiency with which the spin of the accretor can be tidally coupled to the binary orbit to stabilise the mass transfer (Marsh et al. 2004). There has also been a suggestion by Shen (2015) that due to increased friction from ejected material in nova eruptions earlier in the evolution of the binary, all double white dwarf binaries will merge, and that no AM CVn stars should form in this way.

The alternative is that the companion is a core heliumburning star instead of another white dwarf (Savonije et al. 1986; Iben \& Tutukov 1987). Such a binary will reach a minimum period of $\sim 10 \mathrm{~min}$ before mass transfer starts and the helium star will get increasingly degenerate as it evolves to longer periods. 
A third channel involves a hydrogen-rich cataclysmic variable with a donor that is already partially evolved at the onset of mass transfer. The binary then loses its hydrogen through evolution and accretion to become an AM CVn star with a period at the long end of the range. It is considered an unimportant channel compared to the white dwarf and helium star scenarios given the long evolutionary timescales involved, but potential progenitor binaries for this channel have been identified (Breedt et al. 2012; Carter et al. 2013b).

Only the two eclipsing AM CVn stars, YZ LMi (SDSS J0926+3624) and Gaia14aae, have parameters measured to high enough precision to discriminate between these models. YZ LMi is likely to be of helium star origin, although the white dwarf route cannot be ruled out completely (Copperwheat et al. 2011). Gaia14aae on the other hand, is inconsistent with a white dwarf donor scenario, but it is also not straightforwardly compatible with either of the other models (Green et al. 2018b). A detailed discussion of the three formation channels and a review of the observed properties of the AM CVn population is given by Solheim (2010).

A well-defined sample of AM CVn stars from the Sloan Digital Sky Survey (SDSS) made it possible to compare the observed space density of these binaries, $\rho=(5 \pm 3) \times 10^{-7} \mathrm{pc}^{-3}$, to population synthesis predictions (Carter et al. 2013a). Even though the models take into account a range of efficiencies for the spin-orbit coupling and the subsequent AM CVn survival rate, the models overpredict the space density by an order of magnitude. The reason for the discrepancy is not clear, but may be related to the uncertainty of the distribution of these binaries in the Galaxy (Nissanke et al. 2012). Most surveys where AM CVn stars have been found have covered only high Galactic latitudes, and it is possible that a substantial fraction reside in the Galactic plane.

One of the main limitations in modelling the spatial distribution of the AM CVn population and calibrating models of space density and luminosity is the lack of accurate distances. Only five systems have a parallax determined using the Hubble Space Telescope Fine Guidance Sensor (HST FGS; Roelofs et al. 2007b) with distances to others are generally estimated by comparing model fluxes with observations. With reliable distances we can determine the mass transfer rate, $\dot{M}$, which in conjunction with an orbital period, can constrain which of the three formation channels the binary formed. In turn, this can provide a more reliable value for their space density.

The Gaia Data Release 2 (DR2) on 25 April 2018 provided the parallaxes of 1.3 billion stars down to $G \sim 21$ (Gaia Collaboration 2018a) and has allowed us to determine the distances to 41 of the 56 known AM CVn stars. The first Gaia data release (DR1; Gaia Collaboration 2016b) in September 2016 relied on a combined Tycho-Gaia astrometric solution (TGAS; Gaia Collaboration 2016a), and did not include any AM CVn stars. However, it included parallaxes of 16 hydrogen cataclysmic variables (CVs; Ramsay et al. 2017), which provided a validation of the Disc Instability Model which is widely used to model accreting binaries, including AM CVn stars (Osaki 1989; Kotko et al. 2012; Cannizzo \& Nelemans 2015). Another key result from this work was the comparison between the HST FGS, Very Large Array radio data and Gaia DR1 parallax measurements of the CV SS Cyg, which resolved a longstanding discrepancy in the distance to (and hence luminosity of) this system, and showed that the HST parallaxes may be unreliable.

In this paper, we use the GDR2 parallax measurements to determine the absolute magnitudes of AM CVn stars and their mass accretion rates. We then use these results to infer the space density of these binaries.

\section{The known AM CVn stars}

Observationally, AM CVn stars are characterised by their hydrogen deficient optical spectra and blue colour, so surveys for AM CVn stars have typically focussed on these properties to identify new members of the class. The past decade has seen a rapid increase in the number of known AM CVn stars. Firstly because of a dedicated spectroscopic survey of colour-selected targets from SDSS (Carter et al. 2013a, and references therein) and secondly from photometric and spectroscopic follow-up of transient events in large area photometric surveys, such as the Catalina Real-time Transient Survey (CRTS; Breedt et al. 2014), the Palomar Transient Factory (PTF; Levitan et al. 2015) and the All-Sky Automated Survey for Supernovae (ASASSN; Breedt 2015).

Since the data compilation by Levitan et al. (2015), a number of additional AM CVn systems have been discovered. Some of the more recent discoveries include Gaia14aae, the first in which the white dwarf is fully eclipsed (Campbell et al. 2015; Green et al. 2018b), ASASSN-15fp, the longest period AM CVn system to have been observed in outburst so far (Cartier et al. 2017; Marsh et al. 2017), and SDSS J1351-0643, the first system with a period shorter than $17 \mathrm{~min}$ to be discovered in $\sim 15$ years (Green et al. 2018c). In Table 1, we list the 56 AM CVn stars known at present, ordered by increasing orbital period. We provide a full table, including J2000.0 and J2015.5 sky coordinates and multi-wavelength photometry at the CDS. The column description of the full table is shown in Appendix A.

Time series spectroscopy remains the most reliable method to measure the orbital periods of AM CVn stars, but it is a challenging task due to the faintness of many systems and the short exposures which are needed to phase resolve the short orbital period. For systems which display outbursts (see Table 1) the superhump period may be used as a proxy. These are flux variations observed during superoutbursts, resulting from the interaction between the precessing accretion disc and the donor star (e.g. Wood et al. 2011). It is typically a few per cent longer than the orbital period. Other proxies include the relationship between the equivalent width of emission lines and the orbital period (Carter et al. 2013a), and the recurrence time between outbursts (Levitan et al. 2015).

The orbital periods of the known AM CVn stars range from $5.4 \mathrm{~min}$ to $65.6 \mathrm{~min}$. Eight of the known systems do not have an estimate of their orbital period yet. AM CVn stars at the short period end $\left(P_{\text {orb }}<20 \mathrm{~min}\right)$, are akin to novalike CVs, with hot, high state accretion discs. The accretion rate drops as the binary evolves to longer periods, and at the longest periods in the range (i.e. lowest accretion rates) the discs are in a low, stable state. At intermediate periods, $20<P_{\text {orb }} \lesssim 44-52 \mathrm{~min}$, the discs display 1-5 mag outbursts similar to the dwarf nova outbursts observed in the hydrogen cataclysmic variables (Levitan et al. 2011, 2015; Ramsay et al. 2012). The long period boundary below which outbursts are observed is not sharp. For example, Gaia14aae and ASASSN-15fp, with periods of 49.7 and 51.0 min respectively, were discovered as a result of their outbursts, but the long-known system GP Com with $P_{\text {orb }}=46.6 \mathrm{~min}$, has never been observed in outburst. Cannizzo \& Nelemans (2015) show that this is a result of the dependence of the mass transfer rate on the accretor mass, in the sense that systems with a more massive accretor have a higher mass transfer rate at a given orbital period. This dependence is stronger at the long period edge than at the short period edge, resulting in a mix of outbursting and stable systems near $P_{\text {orb }} \sim 44-52 \mathrm{~min}$. 
G. Ramsay et al.: AM CVn stars from Gaia DR2

Table 1. Currently known AM CVn stars ordered by increasing period, either the orbital period (most reliably determined from spectroscopic observations) or the superhump period (sh) which is typically within a few percent longer than the orbital period.

\begin{tabular}{|c|c|c|c|c|c|c|c|c|}
\hline Source & $\begin{array}{l}\text { Period } \\
(\text { mins })\end{array}$ & Outbursts? & $\begin{array}{l}\text { Mag. Range } \\
\text { (filter) }\end{array}$ & Reference & $\begin{array}{r}\text { Parallax } \\
(\text { mas })\end{array}$ & $\begin{array}{r}\sigma \\
\text { (mas) }\end{array}$ & $\begin{array}{r}\text { Distance } \\
(\mathrm{pc}) \\
\end{array}$ & $\begin{array}{r}\sigma \\
(\mathrm{pc}) \\
\end{array}$ \\
\hline $\mathrm{HMCnc}$ & 5.4 & $x$ & 21.1 & 1 & & & & \\
\hline V407 Vul & 9.5 & $x$ & $19.9(V)$ & 2 & 0.095 & 0.327 & 1786 & 667 \\
\hline ES Cet & 10.4 & $x$ & $16.5-16.8$ & 3 & 0.596 & 0.108 & 1584 & 291 \\
\hline SDSS J1351-0643 & 15.7 & $x$ & 18.6 & 43 & 0.596 & 0.313 & 1317 & 531 \\
\hline $\mathrm{AM} \mathrm{CVn}$ & 17.1 & $x$ & 14.2 & 4 & 3.351 & 0.045 & 299.1 & 4.4 \\
\hline SDSS J1908+3940 & 18.1 & $x$ & 16.1 & 5,6 & 0.954 & 0.046 & 1044 & 51 \\
\hline HP Lib & 18.4 & $x$ & $13.6-13.7$ & 7 & 3.62 & 0.05 & 276 & 4 \\
\hline PTF1 J1919+4815 & 22.5 & $\checkmark$ & $18.2-21.8$ & 8 & 0.550 & 0.327 & 1339 & 555 \\
\hline CX361 & 22.9 & $x$ & 17.6 & 9 & 1.016 & 0.146 & 971 & 156 \\
\hline ASASSN-14cc & $22.5(\mathrm{sh})$ & $\checkmark$ & $16.0-20.0(\mathrm{~V})$ & 10 & 0.975 & 0.098 & 1019 & 108 \\
\hline CR Boo & 24.5 & $\checkmark$ & $13.8-17.0$ & 11 & & & & \\
\hline KL Dra & 25.0 & $\checkmark$ & $16.0-19.6$ & 12 & 1.035 & 0.149 & 956 & 153 \\
\hline PTF1 J2219+3135 & $26.1(\mathrm{p})$ & $\checkmark$ & $16.2-20.6$ & 14 & & & & \\
\hline V803 Cen & 26.6 & $\checkmark$ & $12.8-17.0$ & 13,14 & & & & \\
\hline PTF1 J0719+4858 & 26.8 & $\checkmark$ & $15.8-19.4$ & 15 & 1.144 & 0.301 & 861 & 304 \\
\hline ASASSN-15kf & $27.7(\mathrm{sh})$ & $\checkmark$ & $15.0(V)-19.4(\mathrm{~B})$ & 16,17 & & & & \\
\hline YZ LMi (SDSS J0926+3624) & 28.3 & $\checkmark$ & $16.6-19.6$ & 18 & 1.824 & 0.549 & 577 & 324 \\
\hline CP Eri & 28.4 & $\checkmark$ & $16.2-20.2$ & 19 & 0.684 & 0.941 & 964 & 615 \\
\hline SDSS J1043+5632 & $28.5:(\mathrm{p})$ & $\checkmark$ & $17.0-20.3$ & 14 & 0.830 & 0.668 & 979 & 575 \\
\hline CRTS J0910-2008 & $29.7(\mathrm{sh})$ & $\checkmark$ & $14.0-20.4(g)$ & 45 & 0.695 & 0.477 & 1113 & 561 \\
\hline PTF1 J0943+1029 & 30.4 & $\checkmark$ & $16.9(R)-20.7(\mathrm{~g})$ & 20 & & & & \\
\hline CRTS J0105+1903 & 31.6 & $\checkmark$ & $16.3-19.6$ & 21,22 & 1.382 & 0.457 & 734 & 374 \\
\hline PTF1 J1632+3511 & $32.7(\mathrm{p})$ & $\checkmark$ & $17.9-23.0$ & 20 & & & & \\
\hline CRTS J0744+3254 & 33: (p) & $\checkmark$ & $17.4-21.1$ & 14 & & & & \\
\hline V406 Нya & 33.8 & $\checkmark$ & $14.5-19.7$ & 23 & 2.391 & 1.050 & 504 & 493 \\
\hline PTF1J0435+0029 & 34.3 & $\checkmark$ & $18.4(R)-22.3(\mathrm{~g})$ & 20 & & & & \\
\hline SDSS J1730+5545 & 35.2 & $\sim$ & $18.5(V)-20.1$ & $14,24 \mathrm{a}$ & 1.061 & 0.382 & 911 & 420 \\
\hline V558 Vir (2QZ J1427-0123) & $36.6(\mathrm{sh})$ & $\checkmark$ & $15.0-20.5$ & 25 & 1.911 & 1.425 & 677 & 595 \\
\hline SDSS J1240-0159 & 37.4 & $\checkmark$ & $16.8-19.8$ & 27 & 1.857 & 0.611 & 577 & 365 \\
\hline NSV1440 & $37.5(\mathrm{sh})$ & $\checkmark$ & $12.4(V)-17.9(\mathrm{G})$ & 45 & 2.971 & 0.142 & 337 & 17 \\
\hline V744 And (SDSS J0129+3842) & 37.6 & $\checkmark$ & $14.5-19.8$ & $14,26,29$ & 2.066 & 0.529 & 508 & 239 \\
\hline SDSS J1721+2733 & 38.1 & $\checkmark$ & $16.0-20.1$ & 14,31 & 0.798 & 0.665 & 995 & 578 \\
\hline ASASSN-14mv & 41: (sh) & $\checkmark$ & $11.8(V)-18.1(V)$ & $16,17,44$ & 4.069 & 0.119 & 247 & 7 \\
\hline ASASSN-14ei & 43: (sh) & $\checkmark$ & $11.9-17.6(B)$ & 17,33 & 3.92 & 0.045 & 255 & 4 \\
\hline SDSS J1525+3600 & 44.3 & $x$ & 20.2 & 31 & 1.928 & 0.276 & 524 & 90 \\
\hline SDSS J0804+1616 & 44.5 & $\checkmark$ & $17.8-19.0$ & 28,30 & 1.203 & 0.210 & 828 & 173 \\
\hline SDSS J1411+4812 & 46.0 & $\checkmark$ & $12.4-19.7$ & $29,30,48$ & 2.361 & 0.305 & 429 & 65 \\
\hline GPCom & 46.6 & $x$ & $15.9-16.3$ & 32 & 13.731 & 0.060 & 73.0 & 0.4 \\
\hline CRTS J0450-0931 & $47.3(\mathrm{sh})$ & $\checkmark$ & $14.8-20.5$ & 34 & & & & \\
\hline SDSS J0902+3819 & 48.3 & $\checkmark$ & $13.8(V)-20.2(\mathrm{~g})$ & $15,31,35$ & 2.519 & 0.936 & 461 & 435 \\
\hline Gaia14aae & 49.7 & $\checkmark$ & $13.6(V)-18.7(g)$ & 36,49 & 3.871 & 0.155 & 259 & 11 \\
\hline ASASSN-17fp & $51.0(\mathrm{sh})$ & $\checkmark$ & $15.7-20+$ & 37 & & & & \\
\hline SDSS J1208+3550 & 53.0 & $x$ & $18.9-19.4$ & $30,38,39$ & 5.005 & 0.416 & 202 & 18 \\
\hline SDSS J1642+1934 & 54.2 & $x$ & 20.3 & 31,39 & 0.621 & 0.730 & 1044 & 604 \\
\hline SDSS J1552+3201 & 56.3 & $x$ & $20.2-20.6$ & 30,41 & 2.395 & 0.609 & 443 & 227 \\
\hline SDSS J1137+4054 & 59.6: & $x$ & 19.0 & $24 b$ & 4.838 & 0.310 & 209 & 14 \\
\hline V396 Нya & 65.1 & $x$ & 17.6 & 42 & 10.694 & 0.148 & 93.6 & 1.4 \\
\hline SDSS J1319+5915 & 65.6 & $x$ & 19.1 & 40,47 & 4.894 & 0.240 & 205 & 10 \\
\hline PU Aqr (SDSS J2047+0008) & ? & $\checkmark$ & $17.0-24$ & 38 & & & & \\
\hline CRTS J0844-0128 & ? & $\checkmark$ & $17.4-20.3$ & 14 & 0.395 & 0.341 & 1474 & 597 \\
\hline PTF1 J0857+0729 & ? & $\checkmark$ & $18.6-21.8$ & 14 & & & & \\
\hline SDSS J1505+0659 & ? & $x$ & 19.1 & $24 \mathrm{~b}$ & 6.299 & 0.453 & 160 & 12 \\
\hline PTF1 J1523+1845 & ? & $\checkmark$ & $17.6-23.5$ & 14 & & & & \\
\hline ASASSN-14fv & ? & $\checkmark$ & $14.6(V)-20.5(B)$ & 41 & & & & \\
\hline Gaia16all & ? & $\checkmark$ & $16.2-20.6(G)$ & 46 & 0.760 & 0.859 & 956 & 605 \\
\hline SDSS J0807+4852 & ? & $x$ & $19.5(V)-20.4(g)$ & 50 & 0.055 & 2.42 & 883 & 648 \\
\hline
\end{tabular}

Notes. (p) implies the predicted orbital period based on the outburst properties (Levitan et al. 2015). $\checkmark$ or $\boldsymbol{X}$ indicates whether the source has been seen in outburst. The references and full coordinates are given in Appendix A.2. 
Table 2. Gaia DR2 and HST parallaxes of the five AM CVn stars which were measured using the HST Fine Guidance Sensor.

\begin{tabular}{lcc}
\hline \hline Source & $\begin{array}{c}\text { HST } \\
\text { parallax } \\
\text { (mas) }\end{array}$ & $\begin{array}{c}\text { Gaia } \\
\text { parallax } \\
\text { (mas) }\end{array}$ \\
\hline AM CVn & $1.65 \pm 0.30$ & $3.351 \pm 0.045$ \\
HP Lib & $5.07 \pm 0.33$ & $3.622 \pm 0.053$ \\
CR Boo & $2.97 \pm 0.34$ & N/A \\
V803 Cen & $2.88 \pm 0.24$ & N/A \\
GP Com & $13.34 \pm 0.33$ & $13.73 \pm 0.06$ \\
\hline
\end{tabular}

\section{Parallaxes and distances of AM CVns in Gaia DR2}

In Table 1 we show the parallaxes for all AM CVn stars included in the Gaia DR2: out of the 56 known systems 41 have parallax measurements. The closest system is the long known GP Com with a parallax of $13.73 \pm 0.06$ mas, with the most distant being V407 Vul which has a parallax consistent with zero $(0.10 \pm 0.33$ mas $)$. In Table 2 we compare the parallaxes for three sources which have both Gaia and HST parallaxes (V803 Cen and CR Boo have no parallax in Gaia DR2). There is reasonable agreement for GP Com. However, HP Lib is significantly more distant and AM CVn significantly closer compared to the results of HST. Indeed the HST parallax derived for AM CVn implied a greater distance than other estimates and therefore implied a high luminosity and mass accretion rate, all of which were problematic in explaining.

We convert parallaxes from the Gaia DR2 into distances following the guidelines from Bailer-Jones (2015), Astraatmadja \& Bailer-Jones (2016) and Gaia Collaboration (2018c). Given the measured parallax $\varpi$ with the uncertainty $\sigma_{\varpi}$, the probability density of possible values for the distance can be found by using Bayes' theorem:

$P\left(r \mid \varpi, \sigma_{\varpi}\right)=\frac{1}{Z} P\left(\varpi \mid r, \sigma_{\varpi}\right) P(r)$,

where $r$ is the distance, $P\left(\varpi \mid r, \sigma_{\varpi}\right)$ is the likelihood function, that can be assumed Gaussian (Gaia Collaboration 2018b). $P(r)$ is the prior and $Z$ is a normalisation constant. The prior is an arbitrary function that expresses our knowledge on the distribution of the distances of AM CVn stars and allows us to introduce assumptions in the inference procedure that are not related to the measurement of the parallax itself. The properties of various priors and their performance on the data from the Gaia DR1 have been investigated in Astraatmadja \& Bailer-Jones (2016). For this work we adopt the exponentially decreasing space density prior:

$P(r)= \begin{cases}\frac{r^{2}}{2 L^{3}} \exp (-r / L) & \text { if } r>0, \\ 0 & \text { otherwise, }\end{cases}$

where $L$ is the scale length. This prior implies a constant space density of AM CVn stars for $r \ll L$ and an exponential drop for $r \gg 2 L$, where $2 L$ corresponds to the peak of the distribution. The choice of the value for $L$ needs to be fine-tuned to reproduce the distribution of AM CVn stars with the distance. We adopt $L=400 \mathrm{pc}$ calibrated on the mock population of double white dwarf binaries (progenitor systems of AM CVns) from Korol et al. (2017). For more detailed argumentation we refer the reader to Kupfer et al. (2018). In Table 1 we show the inferred distance and uncertainty (which covers the 90 percent confidence interval) for those AM CVn systems which have parallax measurements in the Gaia DR2. The distances range from $73 \mathrm{pc}$ (GP Com) to $1.8 \mathrm{kpc}(\mathrm{V} 407 \mathrm{Vul})$, although the latter is rather uncertain. The median distance is $580 \mathrm{pc}$. For sources closer than $340 \mathrm{pc}$ the mean uncertainty on the distance is $9 \mathrm{pc}$, and for those between $500-1000 \mathrm{pc}$ it is $380 \mathrm{pc}$.

\section{Galactic distribution}

For the 41 systems with parallax and proper motions from Gaia DR2 (shown in Table B.1), we calculate 3D kinematics and put constraints on their population membership: thin disc, thick disc, and halo. Only AM CVn, SDSSJ 1908+3940, CP Eri, SDSS J1730+5545 and SDSS J1240-0159 have measured systemic velocities based on radial velocity curves of their accretion disc lines. Seven additional systems have a strong central spike feature which can be used to measure the radial velocity. These central spike lines are believed to originate close to the photosphere of the accretor and are shifted with the gravitational redshift of the accretor. Assuming an accretor with $M=0.8 M_{\odot}$ and a radius of $R=0.01 R_{\odot}$ leads to a gravitational redshift of $50 \mathrm{~km} \mathrm{~s}^{-1}$, so we correct the measured velocity from the central spike by $50 \mathrm{~km} \mathrm{~s}^{-1}$. For the remaining systems with no measured radial velocity, we assume $0 \mathrm{~km} \mathrm{~s}^{-1}$ with an error of $\pm 50 \mathrm{~km} \mathrm{~s}^{-1}$.

Combined with right ascension and declination, we calculate velocity in the direction of the Galactic Centre $\left(V_{\rho}\right)$ and the Galactic rotation direction $\left(V_{\phi}\right)$, the Galactic orbital eccentricity $(e)$, and the angular momentum in the Galactic $z$ direction $\left(J_{z}\right)$. The Galactic radial velocity $V_{\rho}$ is negative towards the Galactic centre, while stars that are revolving on retrograde orbits around the Galactic Centre have negative $V_{\phi}$. Stars on retrograde orbits have positive $J_{z}$. Thin disc stars generally have very low eccentricities $e$. Population membership can be derived from the position in the $V_{\rho}-V_{\phi}$ diagram and the $J_{z}-e$ diagram following the description in Pauli et al. (2003, 2006).

We show the results in Fig. 1. We find that the most of the systems show a Galactic orbit typical for a thin disc population (see Table B.1). About 10 systems have a typical thick disc orbit. None of the systems show a halo orbit. In a small number of AM CVn stars, the extreme depletion of heavy elements have been taken as evidence that these stars were halo objects (GP Com: Marsh et al. 1991; V396 Hya: Nagel et al. 2009 and PTF1 J0719+4858: Gehron et al. 2014). Our study has shown that these AM CVn stars are likely thin or thick disc objects and not halo objects. It is of interest to understand how disc objects can have such low abundances of heavy elements.

\section{Determining the line of sight extinction}

To determine the absolute magnitude of AM CVn stars we need to subtract the effects of interstellar extinction. Although 47 of the 56 AM CVn stars shown in Table 1 lie at Galactic latitudes $|b|>20^{\circ}$ (implying the extinction is likely to be low), we have determined the line-of-sight extinction to our sources using 3D-dust maps derived from Pan-STARRS1 data (Green et al. 2018a). For each AM CVn star, we derived the extinction, $E_{B-V}$, for the sky co-ordinates and distance of that star given in Table 1 (the uncertainty is typically $E_{B-V} \sim 0.02$ ). To determine the reddening, we use $A_{V}=R \times E_{B-V}$, where we assume the standard value of $R=3.2$. To obtain the reddening in the $g$ band, we assume $A g=1.1 \times A_{V}$ (Cardelli et al. 1989). For those AM CVn stars at declinations too south to feature in the Pan-STARRS1 catalogue we use the dustmaps of Schlafly \& Finkbeiner (2011) 

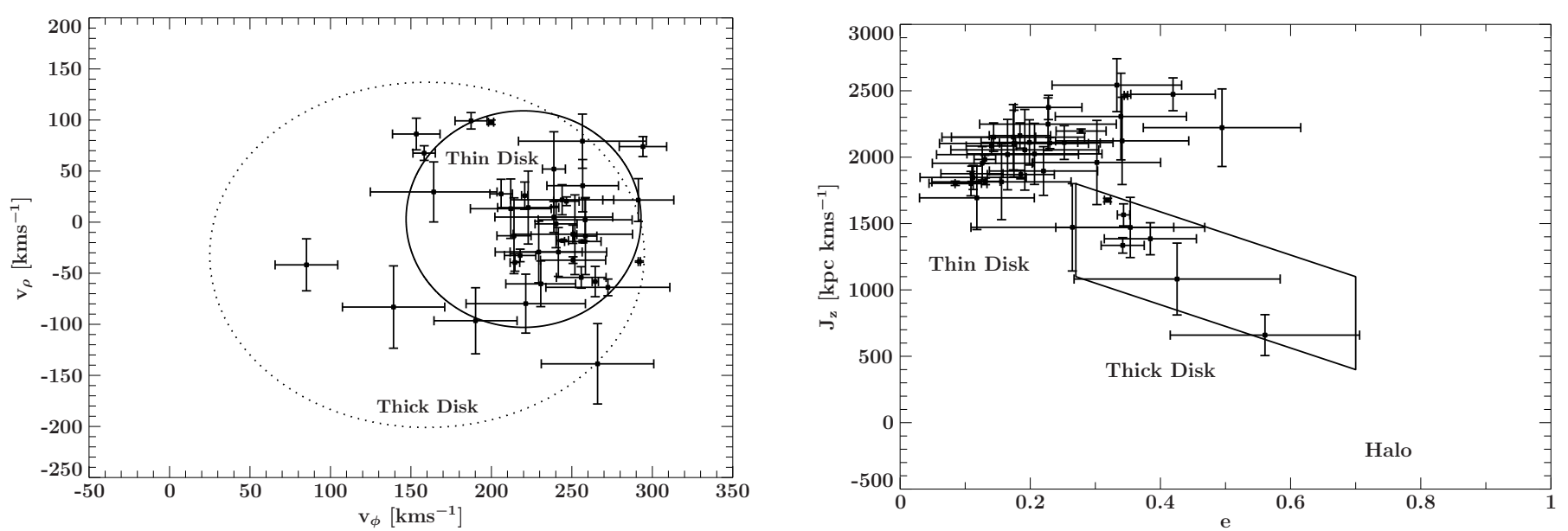

Fig. 1. AM CVn stars in the plane of the velocity in the direction of Galactic rotation $\left(V_{\phi}\right)$ and the Galactic centre $\left(V_{\rho}\right)($ left hand panel $)$ and the orbital eccentricity $(e)$ and the angular momentum in the Galactic $z$ direction $\left(J_{z}\right)$ (right hand panel). The solid and dotted ellipses render the $3 \sigma$ thin and thick disc contours in the $V_{\phi}-V_{\rho}$ diagram, while the solid box in the $e-J_{z}$ marks the thick disc region as specified by Pauli et al. (2006).

which give the extinction to the edge of the Galaxy in that line of sight (this step was required for only two stars and given that the upper limits are only $0.2 \mathrm{mag}$, we do not expect that this uncertainty will significantly effect their place on the period - absolute magnitude relationship).

We show the reddening to those AM CVn stars with parallaxes in Table 3. The median reddening of our sample is $A_{g}=$ $0.14 \mathrm{mag}$, with 90 percent of sources having $A_{g}<0.35 \mathrm{mag}$. Therefore for the majority of our targets the effects of interstellar absorption has a small effect on the resulting absolute magnitudes. The only source with a high extinction, V407 Vul, was previously known to have a high degree of reddening (Motch et al. 1996).

\section{The absolute magnitudes of AM CVn stars}

Using the distances shown in Table 1, we show in Fig. 2 the absolute magnitude, $M_{G}$, against the BP-RP (the "Blue Photometer" and "Red Photometer" colour derived from Gaia data) of AM CVn stars and a sample of Galactic stars with accurate parallax measurements. The single white dwarf track can be seen in the lower left of the figure. High state AM CVn stars are significantly brighter than the isolated white dwarfs, being $M_{G} \sim 6$. The majority of quiescent AM CVn stars appear 1-2 mag brighter than the white dwarfs, being $M_{G} \sim 8-12$, though a minority lie on the white dwarf track.

As the wavelength range of the Gaia $G$ band filter is very broad, it is not the optimal filter with which to test evolutionary models which usually predict the absolute magnitude in the $V$ or $g$ bands. We have therefore collated all the available multifilter photometry of the AM CVn stars from the Pan-STARSS1, Skymapper, GALEX and SDSS surveys and we show these in Appendices A.4-A.7 and associated tables. Since most surveys release mean or median values for each source and filter, the effects of outbursts are generally smoothed out and usually they reflect the quiescent brightness for outbursting systems. In Table 3 we show the mean quiescent $g$ mag which we derive for these systems. For most systems this magnitude is found from analysis of the $g$-band data shown in the appendix. In the case of ASASSN-14cc, a short-period system which is in outburst for around 60 percent of the time, the magnitude measured by Skymapper is representative of its outburst magnitude. We therefore take its quiescent magnitude from Kato et al. (2015).

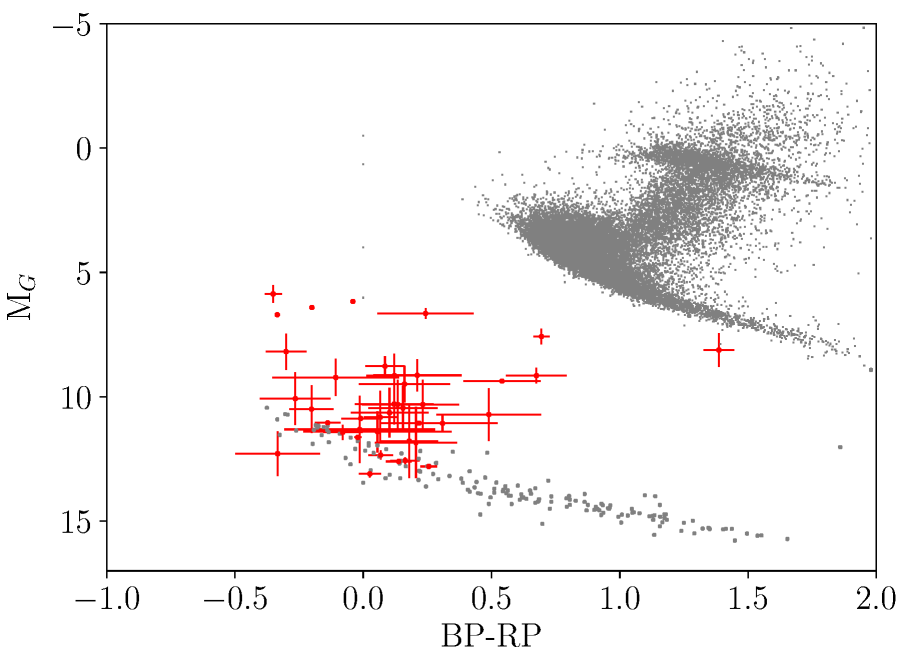

Fig. 2. An HR diagram made using Gaia DR2 data. In red we show the absolute $G$ magnitude, $M_{G}$, as a function of the blue-red colour, BP-RP, for AM CVns with known parallaxes. No reddening or extinction corrections have been applied to this figure. For comparison, in grey points we show a sample of essentially randomly selected main sequence stars which have a parallax better than 1 percent. For single white dwarfs shown as slightly larger grey points) we have used the sample of white dwarfs within 20 pc (Hollands et al. 2018). The majority of AM CVns appear to be brighter than the single white dwarf track, though some long-period systems lie on the track. A clear outlier is V407 Vul, which has $\mathrm{BP}-\mathrm{RP} \approx 1.5$ through a combination of severe reddening and contamination from an unresolved nearby G-star.

Based on these quiescent magnitudes, we calculate the absolute magnitudes shown in Table 3. In Fig. 3 we plot the absolute magnitudes of AM CVn binaries as a function of orbital period, for the 37 systems in which both magnitude and orbital period are known. For those systems which outburst we also plot an approximate outburst magnitude. For comparison, we show the predicted magnitudes of the central white dwarf and the accretion disc, both of which vary in magnitude as a function of orbital period. We show two cooling tracks for a $0.65 M_{\odot}$ central white dwarf, both taken from Bildsten et al. (2006): one assumes a low mass transfer rate (as would be seen for a cool, low-mass white dwarf donor) and one assumes a high mass transfer rate (as would be seen in a hot, high-mass dwarf donor). The donor 
Table 3. Reddening in the $g$ band $\left(A_{g}\right)$, which have been determined using Pan-STARRS1 data (Green et al. 2018a).

\begin{tabular}{|c|c|c|c|c|}
\hline Source & $\begin{array}{r}P_{\text {orb }} \\
(\mathrm{min})\end{array}$ & $\begin{array}{r}A_{g} \\
(\mathrm{mag})\end{array}$ & $\begin{array}{r}g_{q} \\
(\mathrm{mag})\end{array}$ & $\begin{array}{r}M_{g} \\
(\mathrm{mag})\end{array}$ \\
\hline V407 Vul & 9.5 & 4.9 & 20.16 & $4.0 \pm 0.7$ \\
\hline ES Cet & 10.4 & 0.1 & 16.66 & $5.6 \pm 0.4$ \\
\hline SDSS J1351-0643 & 15.7 & 0.1 & 18.66 & $8.0 \pm 0.7$ \\
\hline $\mathrm{AM} \mathrm{CVn}$ & 17.1 & 0.07 & 14.26 & $6.81 \pm 0.03$ \\
\hline SDSS J1908+3940 & 18.1 & 0.53 & 16.16 & $5.5 \pm 0.1$ \\
\hline HP Lib & 18.4 & 0.49 & 13.60 & $5.91 \pm 0.03$ \\
\hline PTF1 J1919+4815 & 22.5 & 0.18 & 20.51 & $9.7 \pm 0.8$ \\
\hline CX361 & 22.9 & 1.16 & 17.48 & $6.4 \pm 0.3$ \\
\hline ASASSN-14cc & 22.5 & 0.12 & 19.0 & $8.9 \pm 0.2$ \\
\hline KL Dra & 25.0 & 0.18 & 18.62 & $8.5 \pm 0.3$ \\
\hline PTF1 J0719+4858 & 26.8 & 0.32 & 19.15 & $9.2 \pm 0.7$ \\
\hline YZ LMi & 28.3 & 0.07 & 19.23 & $10.4 \pm 1.0$ \\
\hline CP Eri & 28.4 & 0.35 & 20.07 & $9.8 \pm 1.1$ \\
\hline SDSS J1043+5632 & 28.5 & 0.07 & 20.35 & $10.33 \pm 1.0$ \\
\hline CRTS J0910-2008 & 30.2 & 0.32 & 19.39 & $9.9 \pm 0.9$ \\
\hline CRTS J0105+1903 & 31.6 & 0.18 & 19.65 & $10.1 \pm 0.9$ \\
\hline V406 I & 3 & 0.11 & 1 & $11.4 \pm 1.5$ \\
\hline SDSS J1730+5545 & 35.2 & 0.14 & 20.05 & $10.1 \pm 0.8$ \\
\hline NSV1440 & 36.3 & $0.21^{m}$ & 18.53 & $10.7 \pm 0.1$ \\
\hline V558 Vir & 36.6 & 0.14 & 19.99 & $10.7 \pm 1.4$ \\
\hline SDSS J1240-0159 & 37.4 & 0.07 & 19.55 & $10.7 \pm 1.1$ \\
\hline V744 And & 37.6 & 0.28 & 19.88 & $11.1 \pm 0.8$ \\
\hline SDSS J1721+2733 & 38.1 & 0.07 & 20.43 & $10.4 \pm 1.0$ \\
\hline ASASSN-14mv & 41 & 0.04 & 17.98 & $11.0 \pm 0.1$ \\
\hline ASASSN-14ei & 43 & $0.21^{m}$ & 15.54 & $8.94 \pm 0.03$ \\
\hline SDSS J1525+3600 & 44.3 & 0.07 & 19.86 & $11.2 \pm 0.3$ \\
\hline SDSS J0804+1616 & 44.5 & 0.18 & 18.60 & $8.8 \pm 0.4$ \\
\hline SDSS J1411+4812 & 46.0 & 0.07 & 19.44 & $11.2 \pm 0.3$ \\
\hline GP Com & 46.6 & 0.00 & 15.92 & $11.60 \pm 0.01$ \\
\hline SDSS J0902+38 & 48.3 & 0.11 & 20.39 & $12.0 \pm 1.4$ \\
\hline Gaia14aae & 49.7 & 0.04 & 18.55 & $11.4 \pm 0.1$ \\
\hline SDSS J1208+3550 & 53.0 & 0.07 & 18.82 & $12.2 \pm 0.2$ \\
\hline SDSS J1642+1934 & 54.2 & 0.25 & 20.35 & $10.0 \pm 1.0$ \\
\hline SDSS J1552+3201 & 56.3 & 0.14 & 20.40 & $12.0 \pm 0.9$ \\
\hline SDSS J1137+4054 & 59.6 & 0.07 & 19.09 & $12.4 \pm 0.1$ \\
\hline V396 Hya & 65.1 & 0.18 & 17.68 & $12.64 \pm 0.03$ \\
\hline SDSS J1319+5915 & 65.6 & 0.04 & 19.12 & $12.6 \pm 0.1$ \\
\hline
\end{tabular}

Notes. Those stars where the reddening has been estimated using the dust maps of (Schlafly \& Finkbeiner 2011) have a superscript $m$ to indicate it is the reddening to the edge of the Galaxy. The third column shows the quiescent $g$ magnitude as determined from the survey magnitudes outlined in the appendix. In the final column we show the absolute magnitude, $M_{g}$. These data are also included in the online table.

masses assumed span the range of white dwarf donor masses predicted by Deloye et al. (2005), but we note that higher-mass donors are possible through other evolutionary channels. The accretion disc magnitudes are calculated by Nelemans et al. (2004).

As Fig. 3 shows, a strong decrease in AM CVn absolute magnitude with increasing orbital period is seen. High-state systems $\left(P_{\text {orb }} \lesssim 20 \mathrm{~min}\right)$ lie close to the predicted accretion disc magnitudes, as expected for these disc-dominated systems. Quiescent systems lie a little above the white dwarf cooling track, with an excess that generally decreases with increasing period, though there is considerable scatter between systems. For outbursting

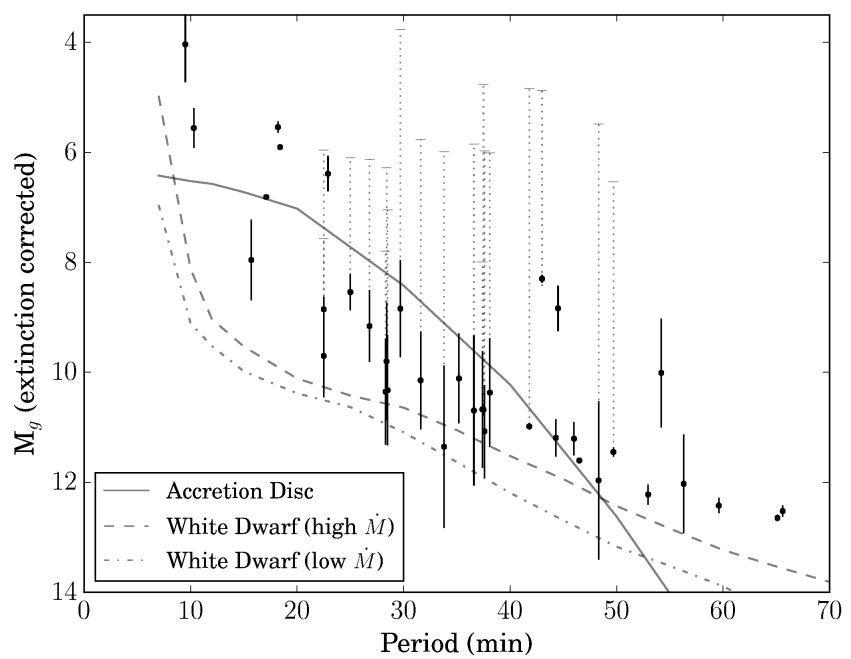

Fig. 3. Extinction-corrected absolute magnitudes $\left(M_{g}\right)$ of $\mathrm{AM} \mathrm{CVn}$ binaries as a function of orbital period. For known outbursting systems, we plot the quiescent magnitude, and show the magnitude change during outburst as a dotted line. The dashed line is the predicted $M_{V}$ of the accretion disc as originally taken from Nelemans et al. (2004) while the solid line shows the evolution of a $0.65 M_{\odot}$ accreting white dwarf with a high-entropy white dwarf donor, including accretion heating, from Bildsten et al. (2006). Most systems appear somewhat brighter than an isolated white dwarf model during quiescence, and close to the dutycycle-scaled disc magnitude during outburst.

systems, the outburst magnitude in most cases is far in excess of the mean disc magnitude.

All AM CVn systems shown in Fig. 3 are brighter than the predicted white dwarf cooling track for a high-mass white dwarf donor, and significantly brighter than the cooling track for a lowmass white dwarf donor. For systems at $P_{\text {orb }}>50 \mathrm{~min}$, which are dominated by their white dwarf magnitudes, this is particularly interesting. It may suggest that AM CVn donors have a systematically higher mass than predicted. Measurements of AM CVn mass ratios imply a similar trend (Green et al. 2018c). If true, this would imply either that white dwarf donors have higher temperatures (and hence higher masses for a given orbital period) than predicted, or that the majority of systems have non-white dwarf donors.

Several individual systems are worthy of note:

- V407 Vul appears unusually bright due to the G star which is co-aligned on the sky. Barros et al. (2007) estimated that the $\mathrm{G}$ star contributes $92 \%$ of the light in the $g$-band, giving a magnitude difference of 2.7 .

- SDSS J1351-0643 has an unexpectedly low magnitude for a high-state system, though its uncertainties are large. This low magnitude could be explained if the system is at a high inclination, such that the disc is viewed close to edge-on. Its broad double-peaked spectrum and photometrically-visible disc precession also support a high inclination.

- ASASSN-14ei, SDSS J0804+1616, Gaia14aae, and SDSS J1642+1934 seem to be unusually bright for their orbital period. For some of these systems there is evidence they may have a high mass transfer rate: SDSS J0804+1616 is unusually variable (Ramsay et al. 2012), while Gaia14aae is among the longest-period outbursting AM CVns and has an unexpectedly high-mass donor star (Campbell et al. 2015; Green et al. 2018b). As discussed in Sect. 7, the elevated magnitude of Gaia14aae is almost entirely due to its hot central white dwarf. 
- Several systems have outburst magnitudes that are lower than the general trend, including PTF1 J1919+4815, YZ LMi, and SDSS J1240-0159. The first two of these systems are partial eclipsers and therefore high-inclination, which will reduce the observed outburst magnitude. The reason for the weak outburst of SDSS J1240-0159, reported by Roelofs (2007), is uncertain.

\section{Component magnitudes of eclipsing AM CVns}

Three AM CVns are currently known which undergo eclipses. In Gaia14aae and YZ LMi, the central white dwarf is eclipsed, while in PTFJ 1919+4815, only the edge of the disc is eclipsed. The first two systems present an opportunity to measure the flux contributions from, and hence absolute magnitudes of, their central white dwarfs. We can additionally measure the flux contribution from the 'bright spot' component of these systems, this being a bright region located at the point of collision between the accretion disc and the stream of infalling matter from the donor.

Photometry of YZ LMi in Sloan $u g r$ bands and of Gaia14aae in ugri bands were presented in Copperwheat et al. (2011) and Green et al. (2018b) respectively. Both papers quote the measured white dwarf fluxes, from which we calculate the absolute magnitudes. To calculate the bright spot contributions, we obtained the best-fit models presented in both papers and measured the bright spot contribution directly. Uncertainties were calculated from the $1-\sigma$ spread of the MCMC results created for those papers when converging on the data. The absolute magnitudes we calculate for these components are presented in Table 4. In the case of YZ LMi, large uncertainties result from the poorly constrained Gaia parallax of the system. The $i$-band Gaia14aae magnitudes are poorly constrained due to the small number of eclipses observed in that band.

\subsection{Central white dwarfs}

These central white dwarf magnitudes may be compared with the predicted white dwarf magnitude track shown in Fig. 3, for corresponding orbital periods. It should be noted that both white dwarfs are somewhat higher mass $\left(\approx 0.85 M_{\odot}\right)$ than the mass assumed in that model $\left(0.65 M_{\odot}\right)$. A larger mass effects the magnitude in two ways: the reduction in size of the white dwarf causes it to appear fainter, but the smaller surface area increases the effect of accretion heating on temperature. With this caveat, the central white dwarf $g$ magnitude of YZ LMi agrees reasonably well with the predicted value of 10.6. For Gaia14aae, the central white dwarf magnitude of 11.66(9) is considerably brighter than the predicted magnitude of 12.4. As shown in Bildsten et al. (2006), the white dwarf mass is less significant than accretion rate for long-period systems like Gaia14aae. It therefore seems likely that the elevation of the central white dwarf magnitude of Gaia14aae above the model track is due to a higher accretion rate than predicted, resulting in a hotter white dwarf.

Given the tightly constrained absolute magnitudes of Gaia14aae, the temperature of the central white dwarf can be estimated. From DB atmosphere models (Bergeron et al. 2011; Tremblay et al. 2011; Kowalski \& Saumon 2006; Holberg \& Bergeron 2006), for a surface gravity $\log (g) \approx 8.5$ as measured by Green et al. (2018b), these magnitudes in all four colour bands predict a temperature of $\approx 17000 \pm 1000 \mathrm{~K}$. This temperature disagrees with the previously established temperature of $12900 \pm 200 \mathrm{~K}$ established from UV flux (Campbell et al
Table 4. Absolute magnitudes of the central white dwarfs and bright spots in two eclipsing systems: YZ LMi and Gaia14aae.

\begin{tabular}{lccc}
\hline \hline Source & Filter & White Dwarf & Bright Spot \\
\hline YZ LMi & $r$ & $10.9(1.0)$ & $14.7(1.6)$ \\
& $g$ & $11.2(1.0)$ & $14.1(1.5)$ \\
& $u$ & $10.7(1.0)$ & $14.5(1.6)$ \\
\hline Gaia14aae & $i$ & $12.17(10)$ & $15.7(1.1)$ \\
& $r$ & $11.95(9)$ & $14.6(2)$ \\
& $g$ & $11.66(9)$ & $14.8(2)$ \\
& $u$ & $11.58(10)$ & $14.4(7)$ \\
\hline
\end{tabular}

2015). The discrepancy may be the result of metals accreted from the donor star, in particular nitrogen, which are expected given the evolved nature of the donor (Nelemans et al. 2010). Such metals would cause absorption in the UV not present in a pure DB atmosphere, making the UV-derived temperature unreliable. We therefore interpret $17000 \mathrm{~K}$ as the most likely temperature of the white dwarf.

\subsection{Bright spots}

The magnitudes of the bright spots in these systems allow for their instantaneous mass transfer rates, $\dot{M}$, to be estimated. If the luminosity of the bright spot is equal to the kinetic energy released by the infalling matter as it slows to match the disc velocity, the luminosity can be described as

$L=\frac{1}{2} \dot{M}\left|\boldsymbol{V}_{\text {stream }}-\boldsymbol{V}_{\text {disc }}\right|^{2}$

where $\boldsymbol{V}_{\text {stream }}$ and $\boldsymbol{V}_{\text {disc }}$ are vectors describing the velocities of material in the stream and disc at the point of intersection between the two. Assuming that the disc material follows a Keplerian orbit and that the stream trajectory is ballistic, these can be calculated based on the measured stellar masses of the system. The luminosity found by Eq. (3) can then be converted to a magnitude using an assumed spectral response for the bright spot. We assume the spectral response of a $12000 \mathrm{~K}$ black-body, as was observed for the cataclysmic variable IP Peg (Marsh 1988). To account for our uncertainty in this choice of spectral response, we increase the uncertainties on the predicted magnitude by $0.2 \mathrm{mag}$, and propagate this through to the uncertainties on $\dot{M}$. We find $\log \left(\dot{M} / M_{\odot} \mathrm{yr}^{-1}\right)=-10.6 \pm 0.4$ for YZ LMi and $-10.74 \pm 0.07$ for Gaia14aae.

For comparison we calculate theoretical values of $\dot{M}$ based on the photometrically measured masses, using the relations in Deloye et al. (2007). It is necessary to assume a value for the donor star's response to mass $\operatorname{loss}, \mathrm{d} \log (R) / \mathrm{d} \log (M)$. For this we assume 0.2, which approximates the tracks in Deloye et al. (2007) for donors evolving isothermally. For YZ LMi, the predicted $\log \left(\dot{M} / M_{\odot} \mathrm{yr}^{-1}\right)=10.0$ is within $1.5-\sigma$ of our measured value. Given that these uncertainties are likely to be non-Gaussian, we do not consider this discrepancy to be significant. In the case of Gaia14aae, an assumed donor response of 0.2 implies $\log \left(\dot{M} / M_{\odot} \mathrm{yr}^{-1}\right)=10.77$, in good agreement with our measured mass transfer rate.

The agreement in the case of Gaia14aae is, to some extent, surprising. Deloye et al. (2007) predict $\mathrm{d} \log (R) / \mathrm{d} \log (M)=0.2$ for AM CVn donors during their short-period evolution. However, once they evolve to periods $\gtrsim 40 \mathrm{~min}$, a change of state in the donor is expected that would result in this value decreasing. 
Table 5. Mass transfer rates determined by fitting the SED of each system using a model consisting of a white dwarf plus steady-state accretion disc model.

\begin{tabular}{lrrrrr}
\hline \hline Source & $\begin{array}{r}\text { Period } \\
(\mathrm{mins})\end{array}$ & $\begin{array}{r}\log 10(\dot{M} \\
\left(M_{\odot} \mathrm{yr}^{-1}\right)\end{array}$ & \pm & $\begin{array}{r}\dot{M} \\
\left(M_{\odot} \mathrm{yr}^{-1}\right)\end{array}$ & \pm \\
\hline ES Cet & 10.4 & -7.61 & 0.46 & $2.5 \times 10^{-8}$ & $1.6 \times 10^{-8}$ \\
SDSSJ1351 & 15.7 & -8.58 & 1.13 & $2.6 \times 10^{-9}$ & $2.4 \times 10^{-9}$ \\
SDSSJ1908 & 18.1 & -6.18 & 0.27 & $6.6 \times 10^{-7}$ & $3.1 \times 10^{-7}$ \\
HP Lib & 18.4 & -8.26 & 0.19 & $5.5 \times 10^{-9}$ & $1.9 \times 10^{-9}$ \\
ASASSN-14cc & 22.5 & -8.63 & 0.14 & $2.3 \times 10^{-9}$ & $6.5 \times 10^{-10}$ \\
ASASSN-14mv & 41.0 & -8.59 & 0.16 & $2.6 \times 10^{-9}$ & $7.9 \times 10^{-10}$ \\
ASASSN-14ei & 43.0 & -10.14 & 0.13 & $7.2 \times 10^{-11}$ & $1.9 \times 10^{-11}$ \\
SDSSJ1525 & 44.3 & -10.15 & 0.46 & $7.1 \times 10^{-11}$ & $4.6 \times 10^{-11}$ \\
SDSSJ1411 & 46.0 & -10.44 & 0.17 & $3.6 \times 10^{-11}$ & $1.2 \times 10^{-11}$ \\
GP Com & 46.6 & -10.64 & 0.25 & $2.3 \times 10^{-11}$ & $1.0 \times 10^{-11}$ \\
Gaia14aae & 49.7 & -10.48 & 0.06 & $3.3 \times 10^{-11}$ & $4.3 \times 10^{-12}$ \\
SDSSJ1208 & 53.0 & -11.15 & 0.15 & $7.1 \times 10^{-12}$ & $2.1 \times 10^{-12}$ \\
SDSSJ1137 & 59.6 & -11.09 & 0.28 & $8.1 \times 10^{-12}$ & $3.9 \times 10^{-12}$ \\
V396 Hya & 65.1 & -10.86 & 0.26 & $1.4 \times 10^{-11}$ & $6.2 \times 10^{-12}$ \\
SDSSJ1319 & 65.6 & -10.94 & 0.26 & $1.1 \times 10^{-11}$ & $5.2 \times 10^{-12}$ \\
\hline
\end{tabular}

Notes. Apart from the eclipsing system (Gaia14aae), we fixed the mass of the white dwarf at $M_{1}=0.8 \pm 0.1 M_{\odot}$ and $\cos (\mathrm{i})=0.5$. We varied radius of the accretion disc to achieve a good fit. The large error the mass transfer rate of SDSS J1505 is due to the large error on the distance.

The fact that 0.2 still appears to hold for Gaia14aae agrees with our suggestion in Green et al. (2018b) that the donor has not yet undergone this change of state.

Recent work by Piersanti et al. (2015) predict the mass accretion rate for systems with periods shorter than $22 \mathrm{~min}$. However, we use the work of Bildsten et al. (2006) who give a relation between the mass transfer rate and the temperature of the accretion-heated central white dwarf in AM CVn systems for the entire orbital period seen in AM CVn stars. Bildsten et al. (2006) predict that, for long-period $(P \gtrsim 30 \mathrm{~min})$ and hence low$\dot{M}$ systems, energy radiated from the white dwarf core will heat the surface more than energy from accretion. The surface temperature will then be higher than would be predicted from $\dot{M}$ alone. This can be seen in Gaia14aae, where the $\dot{M}$ measured above would suggest a black-body white dwarf temperature of $5500 \mathrm{~K}$. The difference between this and our measured temperature of $17000 \mathrm{~K}$ implies, as predicted, that the temperature has decoupled from $\dot{M}$.

\section{Mass transfer rate as a function of orbital period}

One of the reasons that determining the theoretical space density of AM CVn stars has so many uncertainties, is that although there are three likely formation channels, the relative importance of each channel is not well determined. The formation channels largely differ in the nature of the mass donating star. A white dwarf donor would start mass transfer at short orbital periods, whilst the helium star, or highly evolved CV donor, would initially evolve to short orbital periods before evolving to long periods as the mass donating star becomes fully degenerate (see Solheim 2010 for a detailed overview).

If we were able to accurately determine the mass transfer rate for many sources with a range of orbital period we would make progress in understanding the relative importance of the formation channels since they follow different tracks on the $P_{\text {orb }}-\dot{M}$ plane. Determining reliable values for $\dot{M}$ requires photometric information across a wide range of the spectrum, including the $\mathrm{UV}$, where much of the accretion luminosity is emitted (espe- cially for short period systems, for instance, Ramsay et al. 2005, 2006).

For AM CVn stars which have a parallax accurate to within 20 percent, we converted the multi-band photometric data outlined in the appendix to flux units (Jy) (we also included SDSS $\mathrm{J} 1351$ since it has a short orbital period $-15.7 \mathrm{~min}$ - and is therefore interesting from an evolutionary point of view). Some systems, such as KL Dra and AM CVn show a large scatter between the multi-filter flux measurements (possibly due to outbursts in the case of KL Dra, and perhaps significant flickering in the case of AM CVn) and were therefore not suited to determining their mass transfer rate by this means. There were 15 systems which had suitable data and we show their spectral energy distributions in Fig. B.1. For the shortest orbital period systems, the energy distribution is generally still increasing at our bluest flux point (GALEX FUV). For long period systems we can locate the wavelength which the flux appears to peak.

To determine the mass transfer rate we sum up the contribution of flux emitted from the white dwarf and accretion disc. Although the cooling models of Bildsten et al. (2006) assume that the primary star has a mass of $0.65 M_{\odot}$, the primary in both of the eclipsing systems is $M_{1}=0.8 M_{\odot}$. We therefore set $M_{1}=0.8 \pm 0.1 M_{\odot}$ for the non-eclipsing systems. We calculate the radius of the primary using the formula of Eggleton's quoted in Verbunt \& Rappaport (1988). We also assume that the disc is accreting at a steady state and has a number of annuli (40) which we compute the temperature assuming a black-body. Again apart from Gaia14aae the system inclination is not well determined and therefore we fix $\cos (\mathrm{i})=0.5$ (apart from Gaia14aae where $i=86.3^{\circ}$ ). An additional parameter was the radius of the accretion disc, $r_{\text {out }}$. There will be some trade-off between $r_{\text {out }}$ and $\cos (\mathrm{i})$ which we did not explore in detail.

We use modules readily available in python modules including astropy. To determine the mass transfer rate and its uncertainty we randomly select values for distance, mass and extinction within the assigned errors with the other parameters 


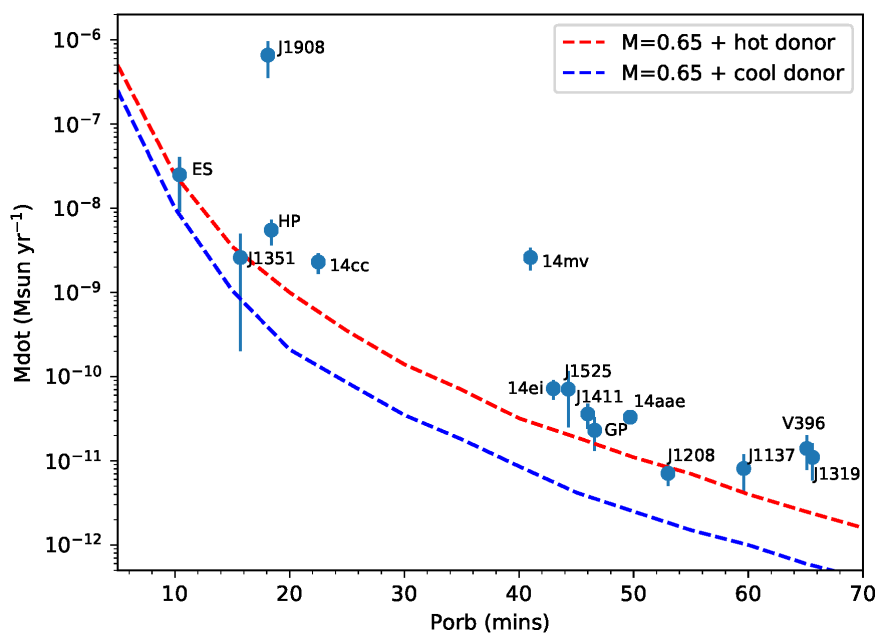

Fig. 4. Predicted mass accretion rate, $\dot{M}$, of Bildsten et al. (2006) as a function of orbital period, where the accretor is a $0.65 M_{\odot}$ white dwarf and the blue track has a cool donor star and the red track a hot donor star. We show the mass transfer rate derived using a white dwarf plus accretion disc model, GDR2 parallax data and multi-wavelength photometric measurements.

kept fixed and repeat this 50 times. From these solutions we determine the mean value and standard uncertainty. Although this is a fairly simple approach the mass transfer rate it is robust enough to make general conclusions from the results as a whole. In Table 5 we show the derived mass transfer rates. In Fig. 4 we show the mass transfer rate as a function of orbital period along with two predicted tracks from Bildsten et al. (2006) and we show the fit to the SED's in Fig. B.1.

In Fig. 4 we can see that some systems appear to lie close to the predicted evolutionary track of a $M_{1}=0.65 M_{\odot}$ and hot donor star. However, there is a tendency for sources to lie above this track. In two cases, SDSS J1908 and ASASSN-14mv, their mass transfer rate is several orders of magnitude greater than the hot donor track. SDSS J1908 lies above the predicted absolute magnitude for its orbital period by $\sim 1$ mag (Fig. 3), which is consistent with a higher mass transfer rate. On the other hand, ASASSN-14mv has an absolute magnitude consistent with expectations, which seems to be at odds with the high mass transfer rate. For the eclipsing system Gaia14aae, we predict a mass transfer rate of $\log 10=-10.48$, which is within $3 \sigma$ of the prediction $(\log 10=-10.74)$ based on using the absolute magnitude of the bright spot (Sect. 7.2). Despite these caveats, our results show evidence that the mass transfer rate for the majority of systems is higher than predicted from the models - and in some cases perhaps by an order of magnitude or more.

The most obvious reason for this result is that the donor star is larger - with a correspondingly higher mass transfer rate - than predicted. Indeed, recent observational work has shown that the donor star is larger than expected from white dwarfs models and in some cases larger than helium star models (Green et al. 2018a). Moreover, a detailed study of the eclipsing system Gaia14aae (Green et al. 2018c) were able to determine system parameters $\left(M_{1}, M_{2}, R_{1}, R_{2}\right)$ to high accuracy. This suggests that this system is likely to have originated via the hydrogen CV evolutionary track. However, no hydrogen is observed in its spectrum as would be expected. These results taken with our findings should provide an impetus to revisiting the formation models.

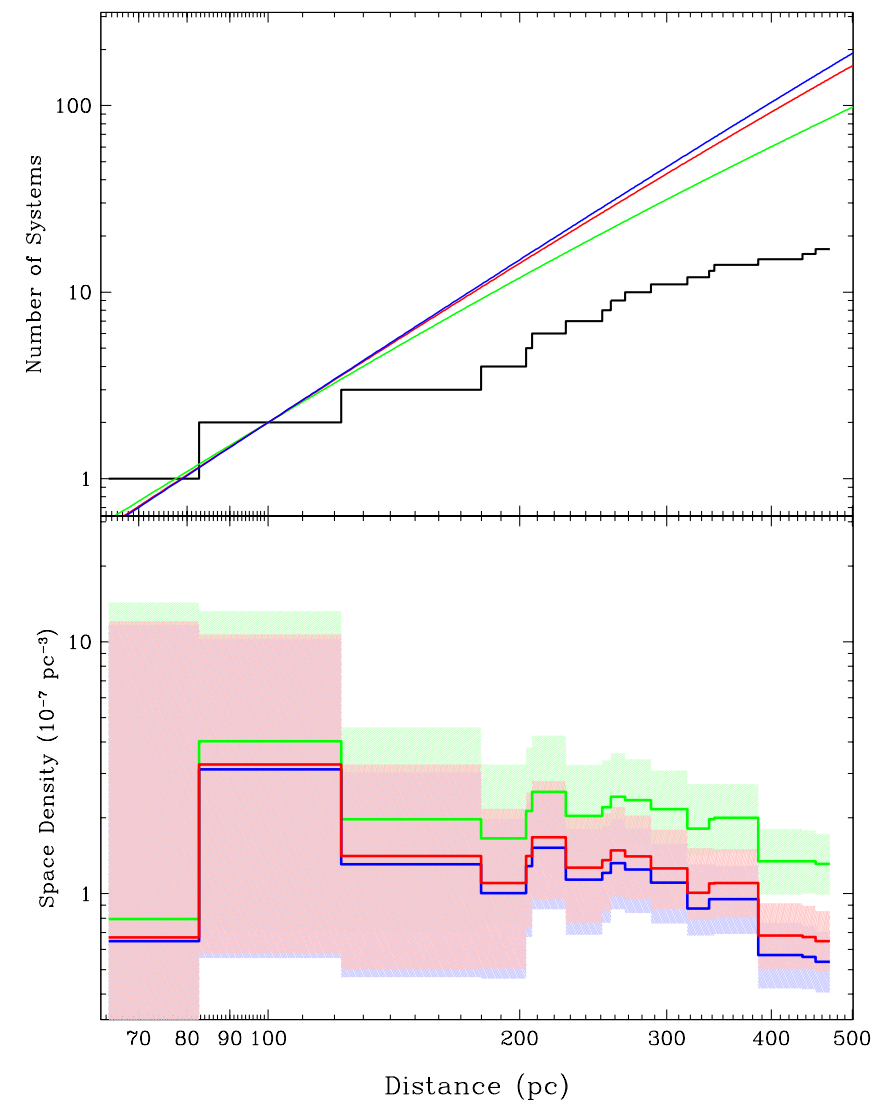

Fig. 5. Top panel: observed and predicted cumulative distribution of AM CVn stars as a function of distance. Predicted distributions are shown for Galactic disc models with three different exponential vertical scale heights: $100 \mathrm{pc}$ (green), $300 \mathrm{pc}$ (red) and $500 \mathrm{pc}$ (blue). Given that AM CVn stars are thought to be preferentially old systems, the two larger scale heights are likely to be more appropriate for this population. All models have been normalised to the data at $100 \mathrm{pc}$. The clear discrepancy between models and data - which increases with distance points to serious incompleteness in the observational sample. Bottom panel: space density (and $1 \sigma$ error bands) as a function of distance, as estimated from the number of AM CVn stars and the effective volume contained within this distance. Effective volumes have been calculated for the same three Galactic disc models as shown in the top panel, thus providing three different space density estimates.

\section{Space density of AM CVn stars}

The AM CVn stars are at the very shortest period end of the binary star orbital period distribution and their space density is a sensitive test for binary evolutionary models. Together with the non-interacting double white dwarf binary, their number largely sets the astrophysical background for gravitational wave detectors such as LISA. Nelemans et al. (2001a) found that the space density of AM CVn stars was very uncertain and subject to uncertainties due to selection effects but determined a range of $0.4-1.7 \times 10^{-4} \mathrm{pc}^{-3}$ (limits being termed "pessimistic" and "optimistic" respectively).

With the advent of the large scale Sloan Digital Sky Survey (SDSS) it was possible to obtain optical spectra of stars which had colours consistent with the known AM CVn stars and therefore identify new systems in a systematic manner. Based on SDSS data (Roelofs et al. 2007a) found a space density of $1-3 \times 10^{-6} \mathrm{pc}^{-3}-$ more than an order of magnitude lower than the pessimistic model of Nelemans et al. (2001b). Using a significantly expanded SDSS sample, Carter et al. (2013a) derived 
Table 6. Results of searching for physically nearby stars to AM CVn stars.

\begin{tabular}{|c|c|c|c|c|c|c|c|c|c|}
\hline & $\begin{array}{c}\text { Gaia } \\
\text { SourceID }\end{array}$ & $\begin{array}{r}\mathrm{RA} \\
\mathrm{J} 2000 \\
\end{array}$ & $\begin{array}{r}\text { Dec } \\
\text { J2000 } \\
\end{array}$ & $\begin{array}{r}\mathrm{p} \\
\text { (mas) }\end{array}$ & $\begin{array}{r}\mathrm{pmra} \\
\left(\mathrm{mas} \mathrm{yr}^{-1}\right)\end{array}$ & $\begin{array}{r}\text { pmdec } \\
\left(\mathrm{mas} \mathrm{yr}^{-1}\right)\end{array}$ & $\begin{array}{r}\mathrm{G} \\
(\mathrm{mag})\end{array}$ & $g-r$ & $\begin{array}{r}\text { Separation } \\
(\mathrm{pc}) \\
\end{array}$ \\
\hline KL Dra & $\begin{array}{l}2239471475135041664 \\
2239448930851818112\end{array}$ & $\begin{array}{l}291.159333 \\
290.953649\end{array}$ & $\begin{array}{l}59.696233 \\
59.610338\end{array}$ & $\begin{array}{l}1.04(15) \\
1.11(33)\end{array}$ & $\begin{array}{l}-2.46(30) \\
-2.89(75)\end{array}$ & $\begin{array}{l}-18.27(26) \\
-18.37(80)\end{array}$ & $\begin{array}{l}19.05 \\
19.44\end{array}$ & $\begin{array}{l}1.21 \\
1.14\end{array}$ & $3.5 \pm 0.6$ \\
\hline SDSS J1525+3600 & $\begin{array}{l}1375131155313563136 \\
1375135415921155968 \\
1375131155313567232\end{array}$ & $\begin{array}{l}231.289887 \\
231.150531 \\
231.288185\end{array}$ & $\begin{array}{l}36.015117 \\
36.107433 \\
36.023225\end{array}$ & $\begin{array}{l}1.93(28) \\
1.63(60) \\
2.28(15)\end{array}$ & $\begin{array}{r}4.79(81) \\
5.25(129) \\
5.24(28)\end{array}$ & $\begin{array}{r}-16.87(81) \\
-16.410(129) \\
-14.492(28)\end{array}$ & $\begin{array}{l}19.665 \\
20.226 \\
18.293\end{array}$ & $\begin{array}{l}0.60 \\
1.13 \\
1.20\end{array}$ & $\begin{array}{r}1.4 \pm 0.3 \\
0.06 \pm 0.01\end{array}$ \\
\hline
\end{tabular}

Notes. The candidate companion stars were selected by searching for stars within $3 \sigma$ of the parallax to the AM CVn star and also $3 \sigma$ of the proper motions in RA and Dec. In the final column we give the physical separation between the star and the AM CVn star.

an observed space density of $5 \pm 3 \times 10^{-7} \mathrm{pc}^{-3}$, a value which is even lower than that of Roelofs et al. (2007a), and is currently the most reliable estimate.

Perhaps the greatest source of uncertainty in predicting the space density lies in the relative importance of the formation channels. As we summarise in Sect. 1, there are three predicted channels - detached double white dwarfs that start mass transfer at very short orbital periods; systems in which a non-degenerate core helium burning star starts mass transfer and binaries in which a white dwarf is accreting from a semi-degenerate star (the hydrogen CV channel). The very low observed space density is compatible with only the double white dwarf contribution to the formation. So either there are many fewer systems coming from the helium star channel (and the CV channel) or we do not sufficiently understand which detached binaries turn into stable mass-transfer systems.

The main challenge in obtaining a space density estimate from the full sample of known AM CVn stars shown in Table 1 is that it contains systems discovered by a variety of different methods and surveys. For example, variability, the presence of emission lines and unusual colours have all been used to detect AM CVn stars. As a result, the sample in Table 1 is neither fluxnor volume-limited, but is instead affected by complex and often poorly understood selection effects. Determining completeness corrections for the entire sample with confidence is therefore impossible.

Nevertheless, the Gaia DR2 distances for the known AM CVn stars do contain crucial information. First, we can test if the sample is complete out to some limiting distance. If so, then a space density estimate can be derived immediately from this volume-limited subsample. Second, even if completeness cannot be established out to any distance, the sample can at least be used to set a firm lower limit on the space density.

Figure 5 shows the results of implementing these ideas. The top panel shows the cumulative distribution of AM CVn stars as a function of distance, compared to the expected distributions for stellar populations associated with the Galactic disc. It is immediately obvious that completeness cannot be established with confidence out to any distance. Based on this data set alone, it is quite possible that there is a significant population of undiscovered AM CVn stars, with systems waiting to be discovered even within $\simeq 100 \mathrm{pc}$.

The bottom panel of Fig. 5 shows the space density one would estimate from the number of $\mathrm{AM} \mathrm{CVn}$ stars as a function of distance, $d$. This is given by $N / V_{\text {eff }}(d)$, where $N$ is the number of systems found at distances smaller than $d$ and $V_{\text {eff }}$ is the effective volume contained within $d$ in a given Galactic model. Since our sample is likely to be incomplete every- where, these estimates must be treated as lower limits. The lower limit suggested by our highest space density estimate would be $\rho_{0}>3 \times 10^{-7} \mathrm{pc}^{-3}$. However, allowing for the Poisson errors affecting our small sample, our best $2 \sigma$ limit is substantially weaker, $\rho_{0}>7 \times 10^{-8} \mathrm{pc}^{-3}$.

\section{Searching for companion stars to the AM CVn stars}

We performed a search to identify companions to those AM CVn stars which have a Gaia DR2 parallax determined to better than 20 percent. For each AM CVn star, we obtained the Gaia DR2 data for all sources within $20^{\prime}$, and then searched for stars which had a parallax and proper motion in RA and DEC within $3 \sigma$. Two AM CVn stars, KL Dra and SDSS J1525+3600, had nearby stars which fulfilled these criteria: their positions and properties are shown in Table 6.

We determined the physical separation between the candidate companion stars and show these in the final column of Table 6. The candidate companion star to KL Dra is $12.4^{\prime}$ distant $(=3.5 \mathrm{pc})$, whilst for SDSSJ1525+3600 they are $8.4^{\prime}$ and $6.1^{\prime \prime}\left(=1.4 \mathrm{pc}\right.$ and $0.06 \mathrm{pc}$ or $\left.1.25 \times 10^{5} \mathrm{AU}\right)$. The more distant candidate companions are too distant to have any effect on the dynamics of the AM CVn binary, although they may have been much closer in the distant past. However, the candidate companion to SDSSJ1525+3600 is only $6.1^{\prime \prime}$ distant providing powerful impetus to obtain a radial velocity measurement to determine whether it is physically associated with the AM CVn binary. If they do show similar velocities then they will be interesting from a binary formation and evolutionary point of view.

\section{Conclusions}

For the first time we have reliable distances to more than a few AM CVn stars. Using these distances we determine the expected cumulative distribution of AM CVn stars and compare it with the distribution of a Galactic disc population. We find that there is likely to be a significant number of AM CVn stars awaiting to be discovered. Since we find that the location of AM CVn stars are in a distinctive region of the HR diagram (Fig. 2) the Gaia DR2 dataset will be a useful tool for identifying candidate systems based on their colour and absolute magnitude.

One of the great uncertainties in predicting the space density of AM CVn stars has been in determining the relative importance of the three formation channels. There is now mounting evidence that the majority donor stars in AM CVn stars are not fully degenerate. We were able to determine the mass transfer rate in $15 \mathrm{AM} \mathrm{CVn}$ stars and find that most have rates which are 
greater than predicted by standard tracks. The donors appear to be larger than expected.

We also find that none of the AM CVn stars which have proper motion and parallax data are likely to be halo objects. Those objects which have very low heavy element abundances are therefore not likely to be due to their age. Coupled with the findings that the parameters of the eclipsing AM CVn star, Gaia14aae, does not readily fit with the models should serve as impetus to revisit these models and the nature of the donor star in particular.

Acknowledgements. This work has made use of data from the European Space Agency (ESA) mission Gaia (https://www.cosmos.esa.int/gaia), processed by the Gaia Data Processing and Analysis Consortium (DPAC, https: //www.cosmos.esa.int/web/gaia/dpac/consortium). Funding for the DPAC has been provided by national institutions, in particular the institutions participating in the Gaia Multilateral Agreement. We extracted GALEX data from Multi-Mission archive at the Space Telescope Science Institute (MAST) The Pan-STARRS1 Surveys (PS1) and the PS1 public science archive have been made possible through contributions by the Institute for Astronomy, the University of Hawaii, the Pan-STARRS Project Office, the Max-Planck Society and its participating institutes, the Max Planck Institute for Astronomy, Heidelberg and the Max Planck Institute for Extraterrestrial Physics, Garching, The Johns Hopkins University, Durham University, the University of Edinburgh, the Queen's University Belfast, the Harvard-Smithsonian Centre for Astrophysics, the Las Cumbres Observatory Global Telescope Network Incorporated, the National Central University of Taiwan, the Space Telescope Science Institute, the Nationa Aeronautics and Space Administration under Grant No. NNX08AR22G issued through the Planetary Science Division of the NASA Science Mission Direc torate, the National Science Foundation Grant No. AST-1238877, the University of Maryland, Eotvos Lorand University (ELTE), the Los Alamos National Laboratory, and the Gordon and Betty Moore Foundation. The national facility capability for SkyMapper has been funded through ARC LIEF grant LE130100104 from the Australian Research Council, awarded to the University of Sydney, the Australian National University, Swinburne University of Technology, the University of Queensland, the University of Western Australia, the University of Melbourne, Curtin University of Technology, Monash University and the Australian Astronomical Observatory. SkyMapper is owned and operated by The Australian National University's Research School of Astronomy and Astrophysics. The survey data were processed and provided by the SkyMapper Team at ANU. The SkyMapper node of the All-Sky Virtual Observatory (ASVO) is hosted at the National Computational Infrastructure (NCI). Development and support the SkyMapper node of the ASVO has been funded in part by Astronomy Australia Limited (AAL) and the Australian Government through the Commonwealth's Education Investment Fund (EIF) and National Collaborative Research Infrastructure Strategy (NCRIS), particularly the National eResearch Collaboration Tools and Resources (NeCTAR) and the Australian National Data Service Projects (ANDS). This research made use of Astropy, a community-developed core Python package for Astronomy (Astropy Collaboration 2018). Armagh Observatory and Planetarium is core funded by the Northern Ireland Executive through the Dept for Communities. MJG acknowledges funding from an STFC studentship via grant number ST/N504506/1. A.A. acknowledges the support of the National Research Council of Thailand (grant number R2561B087).

\section{References}

Abazajian, K. N., Adelman-McCarthy, J. K., Agüeros, M. A., et al. 2009, ApJS, 182,543

Abbott, T. M. C., Robinson, E. L., Hill, G. J., \& Haswell, C. A. 1992, ApJ, 399 680

Anderson, S. F., Haggard, D., Homer, L., et al. 2005, AJ, 130, 2230

Anderson, S. F., Haggard, D., Homer, L., et al. 2008, AJ, 135, 2108

Astraatmadja, T. L., \& Bailer-Jones, C. A. L. 2016, ApJ, 833

Astropy Collaboration (Price-Whelan, A. M., et al.) 2018, AJ, 156, 123

Bailer-Jones, C. A. L. 2015, PASP, 127, 119

Barros, S. C. C., Marsh, T. R., Dhillon, V. S., et al. 2007, MNRAS, 374, 1334

Bergeron, P., Wesemael, F., Dufour, P., et al. 2011, ApJ, 737, 28

Bessell, M., Bloxham, G., Schmidt, B., et al. 2011, PASP, 123, 789

Bianchi, L. 2014, Ap\&SS, 354, 103

Bildsten, L., Townsley, D. M., Deloye, C. J., \& Nelemans, G. 2006, ApJ, 640, 446

Breedt, E. 2015, Proc. Sci., 255, 25

Breedt, E., Gänsicke, B. T., Marsh, T. R., et al. 2012, MNRAS, 425, 2548

Breedt, E., Gänsicke, B. T., Drake, A. J., et al. 2014, MNRAS, 443, 3174
Campbell, H. C., Marsh, T. R., Fraser, M., et al. 2015, MNRAS, 452, 1060 Cannizzo, J. K., \& Nelemans, G. 2015, ApJ, 803, 19

Cardelli, J. C., Clayton, G. C., \& Mathis, J. S. 1989, ApJ, 345, 245

Carter, P. J., Marsh, T. R., Steeghs, D., et al. 2013a, MNRAS, 429, 2143

Carter, P. J., Steeghs, D., de Miguel, E., et al. 2013b, MNRAS, 431, 372

Carter, P. J., Steeghs, D., Marsh, T. R., et al. 2014a, MNRAS, 437, 2894

Carter, P. J., Gänsicke, B. T., Steeghs, D., et al. 2014b, MNRAS, 439, 2848

Cartier, R., Gutierrez, C. P., Smith, M., et al. 2017, ATel, 10334

Chambers, K. C., Magnier, E. A., Metcalfe, N., et al. 2016, ArXiv e-prints [arXiv:1612.05560]

Copperwheat, C. M., Marsh, T. R., Littlefair, S. P., et al. 2011, MNRAS, 410, 1113

Deloye, C. J., Bildsten, L., \& Nelemans, G. 2005, ApJ, 624, 934

Deloye, C. J., Taam, R. E., Winisdoerffer, C., \& Chabrier, G. 2007, MNRAS, 381,525

Espaillat, C., Patterson, J., Warner, B., \& Woudt, P. 2005, PASP, 117, 189

Fontaine, G., Brassard, P., Green, E. M., et al. 2011, ApJ, 726, 92

Gaia Collaboration (Brown, A. G. A., et al.) 2016a, A\&A, 595, A2

Gaia Collaboration (Prusti, T., et al.) 2016b, A\&A, 595, A1

Gaia Collaboration (Brown, A. G. A., et al.) 2018a, A\&A, 616, A1

Gaia Collaboration (Lindegren, L., et al.) 2018b, A\&A, 616, A2

Gaia Collaboration (Luri, X., et al.) 2018c, A\&A, 616, A9

Gehron, K., Nagel, T., Rauch, T., \& Werner, K. 2014, A\&A, 562, A132

Green, G. M., Schlafly, E. F., Finkbeiner, D., et al. 2018a, MNRAS, 478, 651

Green, M. J., Marsh, T. R., Steeghs, D. T. H., et al. 2018b, MNRAS, 476, 1663

Green, M. J., Hermes, J. J., Marsh, T. R., et al. 2018c, MNRAS, 477, 5646

Holberg, J. B., \& Bergeron, P. 2006, AJ, 132, 1221

Hollands, M. A., Tremblay, P.-E., Gänsicke, B. T., Gentile-Fusillo, N. P., \&

Toonen, S. 2018, MNRAS, 480, 3942

Iben, I. J., \& Tutukov, A. V. 1987, ApJ, 313, 727

Kato, T., Stubbings, R., Monard, B., et al. 2004, PASJ, 56, S89

Kato, T., Ohshima, T., Denisenko, D., et al. 2014, PASJ, 66, L7

Kato, T., Hambsch, F.-J., \& Monard, B. 2015, PASJ, 67, L2

Kepler, S. O., Pelisoli, I., Koester, D., et al. 2015, MNRAS, 446, 4078

Kong, X., Luo, A. L., Li, X. R., et al. 2018, PASP, 130, 084203

Korol, V., Rossi, E. M., Groot, P. J., et al. 2017, MNRAS, 470, 1894

Kotko, I., Lasota, J.-P., Dubus, G., \& Hameury, J.-M. 2012, A\&A, 544, A13

Kowalski, P. M., \& Saumon, D. 2006, ApJ, 651, L137

Kupfer, T., Groot, P. J., Levitan, D., et al. 2013, MNRAS, 432, 2048

Kupfer, T., Groot, P. J., Bloemen, S., et al. 2015, MNRAS, 453, 483

Kupfer, T., Korol, V., Shah, S., et al. 2018, MNRAS, 480, 302

Levitan, D., Fulton, B. J., Groot, P. J., et al. 2011, ApJ, 739, 68

Levitan, D., Kupfer, T., Groot, P. J., et al. 2013, MNRAS, 430, 996

Levitan, D., Kupfer, T., Groot, P. J., et al. 2014, ApJ, 785, 114

Levitan, D., Groot, P. J., Prince, T. A., et al. 2015, MNRAS, 446, 391

Marsh, T. R. 1988, MNRAS, 231, 1117

Marsh, T. R., Horne, K., \& Rosen, S. R. 1991, ApJ, 366, 535

Marsh, T. R., Nelemans, G., \& Steeghs, D. 2004, MNRAS, 350, 113

Marsh, T., Parsons, S., \& Dhillon, V. 2017, ATel, 10354

Martin, D. C., Fanson, J., Schiminovich, D., et al. 2005, ApJ, 619, L1

Motch, C., Haberl, F., Guillout, P., et al. 1996, A\&A, 307, 459

Motsoaledi, M. 2015, Proc. Sci., 255, 16

Nagel, T., Rauch, T., \& Werner, K. 2009, A\&A, 499, 773

Nather, R. E., Robinson, E. L., \& Stover, R. J. 1981, ApJ, 244, 269

Nelemans, G., Portegies Zwart, S. F., Verbunt, F., \& Yungelson, L. R. 2001a, A\&A, 368, 939

Nelemans, G., Steeghs, D., \& Groot, P. J. 2001b, MNRAS, 326, 621

Nelemans, G., Yungelson, L. R., \& Portegies Zwart, S. F. 2004, MNRAS, 349 181

Nelemans, G., Yungelson, L. R., van der Sluys, M. V., \& Tout, C. A. 2010, MNRAS, 401, 1347

Nissanke, S., Vallisneri, M., Nelemans, G., et al. 2012, ApJ, 758, 131

O'Donoghue, D., Kilkenny, D., Chen, A., et al. 1994, MNRAS, 271, 910

Osaki, Y. 1989, PASJ, 41, 10050

Paczyński, B. 1967, Acta Astron., 17, 287

Pauli, E.-M., Napiwotzki, R., Altmann, M., et al. 2003, A\&A, 400, 877

Pauli, E.-M., Napiwotzki, R., Heber, U., Altmann, M., \& Odenkirchen, M. 2006, A\&A, 447, 173

Piersanti, L., Yungelson, L. R., \& Tornambé, A. 2015, MNRAS, 452, 2897

Prieto, J. L., Morrell, N., Grupe, D., et al. 2014, ATel, 6475

Ramsay, G., Hakala, P., \& Cropper, M. 2002a, MNRAS, 332, L7

Ramsay, G., Wu, K., Cropper, M., et al. 2002b, MNRAS, 333, 575

Ramsay, G., Hakala, P., Marsh, T., et al. 2005, A\&A, 440, 675

Ramsay, G., Groot, P. J., Marsh, T., et al. 2006, A\&A, 457, 623

Ramsay, G., Barclay, T., Steeghs, D., et al. 2012, MNRAS, 419, 2836 
Ramsay, G., Schreiber, M., Gänsicke, B. T., \& Wheatley, P. J. 2017, A\&A, 604A, 107

Rau, A., Roelofs, G. H. A., Groot, P. J., et al. 2010, ApJ, 708, 456

Roelofs, G. H. A. 2007, PhD Thesis, University of Nijmegen, The Netherlands

Roelofs, G. H. A., Groot, P. J., Marsh, T. R., et al. 2005, MNRAS, 361, 487

Roelofs, G. H. A., Nelemans, G., \& Groot, P. J. 2007a, MNRAS, 382, 685

Roelofs, G. H. A., Groot, P. J., Benedict, G. F., et al. 2007b, ApJ, 666, 1174

Roelofs, G. H. A., Groot, P. J., Steeghs, D., et al. 2009, MNRAS, 394, 367

Ruiz, M. T., Rojo, P. M., Garay, G., \& Maza, J. 2001, ApJ, 552, 679

Savonije, G. J., de Kool, M., \& van den Heuvel, E. P. J. 1986, A\&A, 155,51

Scalzo, R. A., Yuan, F., Childress, M. J., et al. 2017, PASA, 34, 30

Schlafly, E. F., \& Finkbeiner, D. P. 2011, ApJ, 737, 103

Shears, J., Brady, S., Koff, R., Goff, W., \& Boyd, D. 2012, J. Br. Astron. Assoc., 122,49
Shen, K. J. 2015, ApJ, 805, L6

Solheim, J.-E. 2010, PASP, 122, 1133

Thorstensen, J. R., \& Skinner, J. N. 2012, AJ, 144, 81

Tonry, J. L., Stubbs, C. W., Lykke, K. R., et al. 2012, ApJ, 750, 99

Tremblay, P.-E., Bergeron, P., \& Gianninas, A. 2011, ApJ, 730, 128

Verbunt, F., \& Rappaport, S. 1988, ApJ, 332, 193

Wagner, R. M., Kaur, A., Porter, A., et al. 2014, ATel, 6669

Wevers, T., Torres, M. A. P., Jonker, P. G., et al. 2016, MNRAS, 462, L106

Wood, M. A., Winget, D. E., Nather, R. E., et al. 1987, ApJ, 313, 757

Wood, M. A., Casey, M. J., Garnavich, P. M., \& Haag, B. 2002, MNRAS, 334, 87

Wood, M. A., Still, M. D., Howell, S. B., et al. 2011, ApJ, 741, 105

Wood-Vasey, W. M., Aldering, G., Nugent, P., \& Li, K. 2003, IAUC, 8077

Woudt, P. A., Warner, B., \& Rykoff, E. 2005, IAUC, 8531

Woudt, P. A., Warner, B., \& Motsoaledi, M. 2013, ATel, 4726

York, D. G. 2000, AJ, 120, 1579 


\section{Appendix A: Photometry of AM CVn stars: online table}

We provide an online table in fits format ${ }^{1}$ which includes data on all of the known AM CVn stars. The columns and caveats are described below.

\section{A.1. Target names and coordinates}

In the main body of the paper (e.g. Table 1), we use IAU Variable Star names or shortened survey IDs for each of the targets. The online table includes these IDs (Col. 1), as well as alternative names by which each target is known (Col. 2).

We give the J2015.5 coordinates from Gaia where available (Cols. 5, 6), as well as ICRS J2000.0 coordinates for all sources (Cols. 3, 4). The J2000.0 coordinates were derived from the Gaia 5-parameter astrometry where available, otherwise the table shows the most precise coordinates available for that target. We also computed the IAU 1958 Galactic latitude and longitude for each target. These are given in Cols. 7 and 8.

The Gaia DR2 source ID is shown in Col. 9.

\section{A.2. Orbital period and brightness range}

Columns 10 and 11 show the period of the binary in days and minutes respectively, with a flag/comment in Col. 12 to indicate whether the period is the orbital period ("orb", most reliably measured from time series spectroscopy), the superhump period ("sh", resulting from disc precession during the outburst and typically a few per cent longer than the orbital period), estimated from the outburst recurrence time ("est rec", Levitan et al. 2015) or whether no period has been measured for the system so far ("unknown"). References for the discovery and the period measurements are indexed in Col. 15, corresponding to the following references:

[1] Ramsay et al. 2002a, [2] Ramsay et al. 2002b, [3] Espaillat et al. 2005, [4] Nelemans et al. 2001b, [5] Fontaine 2011, [6] Kupfer et al. 2015, [7] O'Donoghue et al. 1994, [8] Levitan et al. 2014, [9] Wevers et al. 2016, [10] Kato et al. 2015, [11] Wood et al. 1987, [12] Wood et al. 2002, [13] Kato et al. 2004, [14] Levitan et al. 2015, [15] Levitan et al. 2011, [16] ASASSN http://www.astronomy . ohio-state.edu/ assassin/transients.html, [17] AAVSO https://www . aavso.org, [18] Copperwheat et al. 2011, [19] Abbott et al. 1992, [20] Levitan et al. 2013, [21] Thorstensen \& Skinner 2012, [22] Motsoaledi 2015, [23] Wood-Vasey et al. 2003, [24a] Carter et al. 2014a, [24b] Carter et al. 2014b, [25] Woudt et al. 2005, [26] Shears et al. 2012, [27] Roelofs et al. 2005, [28] Roelofs et al. 2009, [29] Anderson et al. 2005, [30] Ramsay et al. 2012, [31] Rau et al. 2010, [32] Nather et al. 1981, [33] Prieto et al. 2014, [34] Woudt et al. 2013, [35] Kato et al. 2014, [36] Campbell et al. 2015, [37] Marsh et al. 2017, [38] Anderson et al. 2008, [39] Kupfer et al. 2013, [40] Kepler et al. 2015, [41] Wagner et al. 2014, [42] Ruiz et al. 2001, [43] Green et al. 2018c, [44] Kato, vsnet-alert 18124, [45] Aungwerojwit et al. (in prep.), [46] Breedt et al. (in prep.), [47] Kupfer et al. (in prep.), [48] Maehara \& Kojima, vsnet-alert 22174, [49] Green et al. 2018b, [50] Kong et al. 2018.

The $\mathrm{Y} / \mathrm{N}$ flag in Col. 13 indicates whether the system has been observed in outburst, and the magnitude range (with filter information) is given in Col. 14 .

\section{A.3. Parallax, reddening and derived quantities}

The calculation of the following quantities were discussed in detail in the main text:

Columns 16-17: parallax and error (mas), taken from Gaia DR2.

Columns 18-19: distance and error (pc) - calculated from an exponentially decreasing space density prior with $L=400 \mathrm{pc}$. See Sect. 3.

Columns 20-21: extinction and reddening parameters $E(B-V)$ and $A_{g}$ towards each system (mag), derived from the PanSTARRS1 distance reddening maps (Green et al. 2018a) as discussed in Sect. 5. ASASSN-14cc, ASASSN-14ei, NSV1440 and Gaia16all have parallax measurements from Gaia, but are not in the Pan-STARRS1 footprint. For these four stars we show the maximum reddening from Schlafly \& Finkbeiner (2011). A note has been added in Col. 66 of the table.

Column 22: $g$ band quiescent magnitude taken from PanSTARRS, used to calculated the absolute magnitude.

Columns 23-24: absolute magnitude $M_{g}$ and its associated error (mag).

\section{A.4. GALEX}

Although Swift and XMM-Newton has been used to observe some AM CVn stars, GALEX provided the opportunity to take an unbiased snapshot survey of their near UV flux. GALEX was launched in April 2003 and had a lifetime of ten years and performed observations at UV wavelengths (Martin 2005). It had a primary mirror $50 \mathrm{~cm}$ in diameter and had a field of view of $1.25^{\circ}$. It had two UV channels, the Near UV (NUV, central wavelength $2271 \AA$ ) and the Far UV (FUV, $1528 \AA$ ), the latter failing after 6 years. Whilst it could perform relatively short pointed observations, it also carried out a wide field survey and by its end it had sampled 3/4 of the sky in one UV band (Bianchi 2014). 41 of the known AM CVn stars are included in the catalogue of Bianchi (2014). We show the FUV and NUV fluxes in units of mJy in Cols. 25-26 (FUV) and Cols. 27-28 (NUV) of the online table.

\section{A.5. SDSS}

The Sloan Digital Sky Survey (SDSS) uses a $2.5 \mathrm{~m}$ telescope at Apache Point Observatory in New Mexico, and has a field of view three degrees across (York 2000). Its Data Release 7 includes a photometric catalogue of northern-hemisphere targets (Abazajian et al. 2009).

We performed a cross-match of known AM CVn J2000.0 positions with all SDSS photometric objects, using a search radius of 2 arcsec. We identified 34 AM CVns which have SDSS photometry. All but two of these magnitudes are consistent with the faint end of the range shown in Table 1. The two outliers are SDSS J0804+1616, which is brighter by approximately $0.7 \mathrm{mag}$ (perhaps consistent with the largescale variability this system shows even in quiescence; Roelofs et al. 2009) and PU Aqr=SDSS J2047+0008, which is brighter by approximately $1.8 \mathrm{mag}$ and may have been in outburst or in decline from outburst when the SDSS data were collected.

The SDSS ugriz magnitudes and their errors are shown in columns $29-38$ of the online table.

\footnotetext{
1 Available at the CDS and at https://www.ast.cam.ac.uk/ ebreedt/web/AMCVn_datatable_Ramsay_etal_2018.fits
} 
Table A.1. PanSTARRS1 measurements of these three AM CVn stars appears to have been taken in different accretion states in the different bands.

\begin{tabular}{lccccc}
\hline \hline Source & $g$ & $r$ & $i$ & $z$ & $y$ \\
\hline CR Boo & $16.630(42)$ & $14.406(130)$ & $14.965(92)$ & $15.154(142)$ & $15.494(96)$ \\
$\quad$ range & 16.61 .16 .78 & $14.12 . .16 .74$ & $14.56 . .15 .65$ & $14.47 . .17 .07$ & $15.27 . .17 .41$ \\
SDSS J0804+1616 & $18.222(61)$ & $18.432(44)$ & $18.556(26)$ & $18.639(33)$ & $18.493(29)$ \\
$\quad$ range & $18.09 . .18 .60$ & $18.21 . .18 .60$ & $18.50 . .18 .77$ & $18.50 . .18 .86$ & $18.25 . .18 .92$ \\
CRTS J0744+3254 & $21.345(275)$ & $18.884(21)$ & $21.397(55)$ & $17.803(23)$ & $18.298(16)$ \\
$\quad$ range & 21.32 .21 .87 & $18.81 . .18 .91$ & $21.30 . .21 .50$ & $17.81 . .17 .89$ & $18.29 . .18 .32$ \\
\hline
\end{tabular}

Notes. In addition to the magnitudes shown in the online table (and repeated here for clarity), we also show the range in magnitude for the detections in each filter.

\section{A.6. Pan-STARRS1}

Pan-STARRS1 is a $1.8 \mathrm{~m}$ telescope with a field of view of seven square degrees, located on Halakala Observatory in Hawaii. There are five broad-band filters which are close to, but not exactly, the SDSS filters grizy (Chambers et al. 2016; Tonry 2012). It is performing an all-sky survey with declinations $>-30^{\circ}$.

We performed a cross-match with Pan-STARRS1 DR1 using a 2 arcsec match radius to account for uncertainty in the recorded positions of the AM CVn stars. The mean AB PSF grizy magnitudes are shown in columns 39-48 of the online table for all systems which were found to have a match.

For those AM CVn stars which do not show outbursts the mean $g$ mag is consistent with the values shown in Table 1. For those systems which do show outburst, Pan-STARRS1 has a mean magnitude which is consistent with the source being in quiescent apart from three systems: CR Boo where $g$ is more consistent with it being in quiescent and the other filters in outburst; SDSS J0804+1616 has range in magnitude in Table 1 between 17.8-19.0 and the mean Pan-STARRS1 mag appears in the middle of this range and CRTS J0744+3254 which has a PanSTARRS $1 g$ mag much fainter than $r$. The Pan-STARRS1 DR1 gives a mean magnitude of the source over the pointings covered. However, it also gives a range in magnitudes for the different filters. For the three sources which show evidence for being in different accretion states, we show in Table A. 1 the range in these filters' measurements.

\section{A.7. Skymapper}

Skymapper is a telescope with a $1.35 \mathrm{~m}$ primary mirror located Siding Spring Observatory, Australia, and has a camera which covers $2.4 \times 2.3$ degrees of the sky (Scalzo 2017). Its primary goal is to obtain a map of the southern sky in a number of filters, with periods of poor conditions being focussed on detecting transients. There are six filters, with the bluest $u, v$, being narrow band filters ( $F W H M=42$ and $28 \mathrm{~nm}$ respectively) which cover the SDSS $u$ band. The filters griz differ compared to their SDSS equivalents by $40 \mathrm{~nm}$ in effective wavelength and width (Bessell et al. 2011).

We performed a 2 arcsec radius cross-match with the Skymapper DR1.1 release and find that six AM CVn stars have at least one detection in one filter. We show the magnitudes in the uvgrzi filters on the AB mag scale in columns 49-60 of the online table.

\section{A.8. Kinematics}

Columns 61-64 list the proper motions in RA and Dec (mas) from Gaia DR2. The population membership (thick/thin disc of the Galaxy), as computed in Sect. 4, is shown in Col. 65. For convenience, these data are also shown in Table B.1.

The final column in the online table, 66 , is a text field for specific notes about the data for that target. 


\section{Appendix B: SEDs and kinematic data}

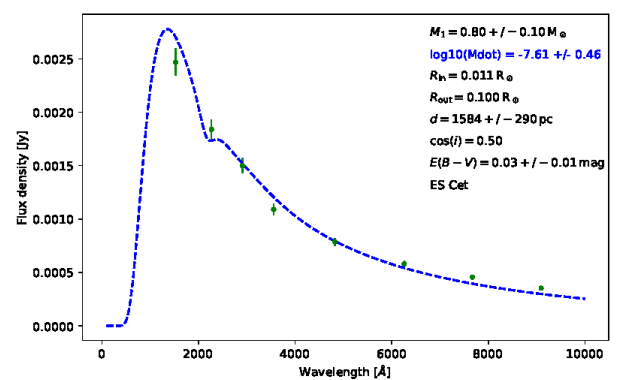

(a)

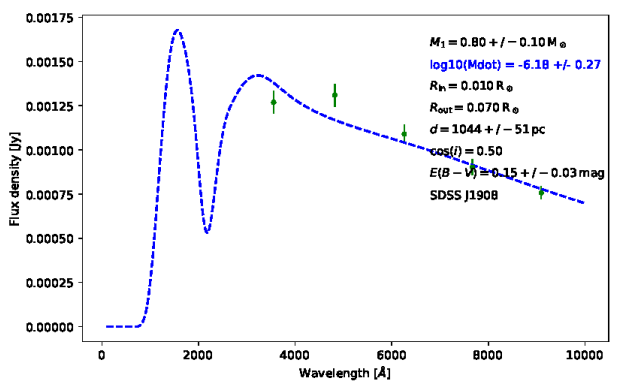

(c)

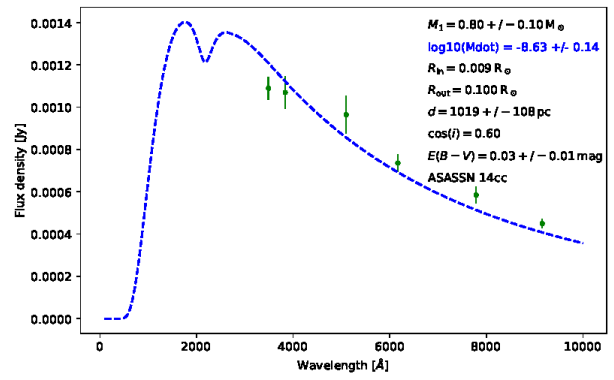

(e)

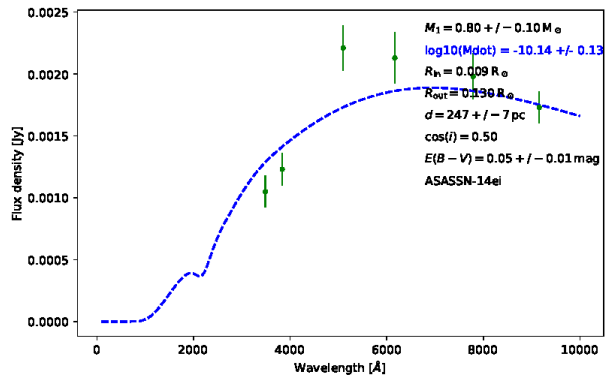

(g)

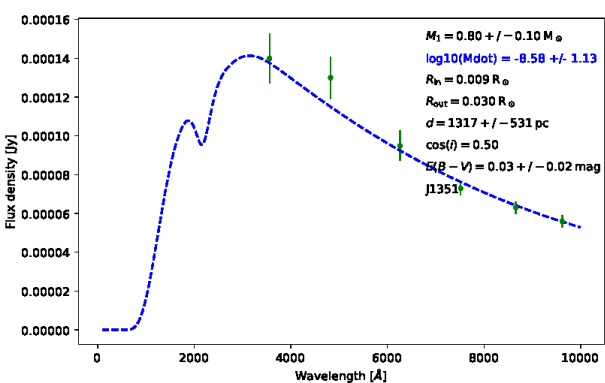

(b)

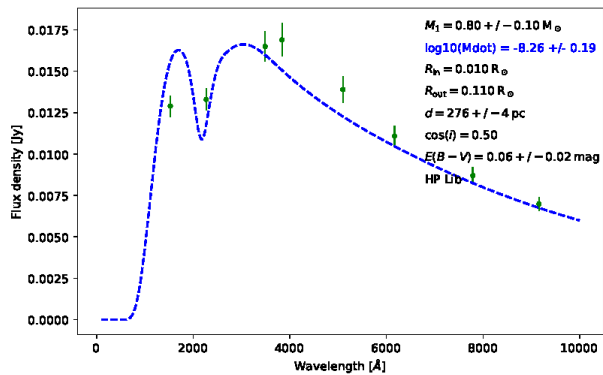

(d)

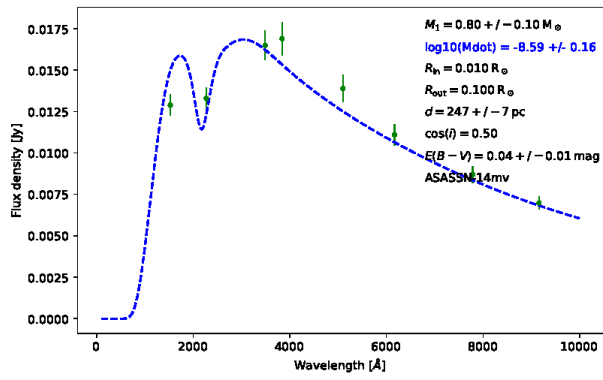

(f)

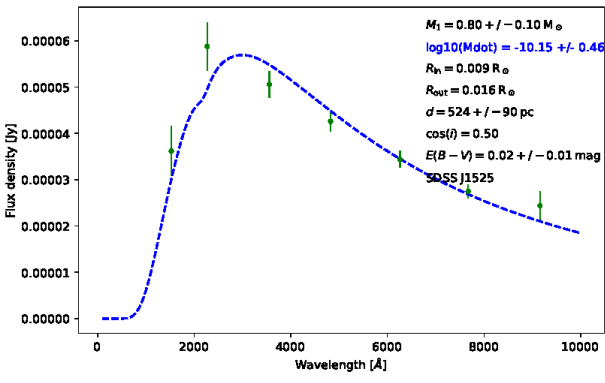

(h)

Fig. B.1. Spectral energy distribution of AM CVn stars which have a parallax accurate to within 20 percent and have multi-colour survey data in more than two filter passbands. In the solid line we show the model spectral energy distribution where $M_{1}, E_{B-V}$ and distance are restricted to the values shown in each panel and the mass transfer rate, $\dot{M}$, is a free parameter. 
A\&A 620, A141 (2018)

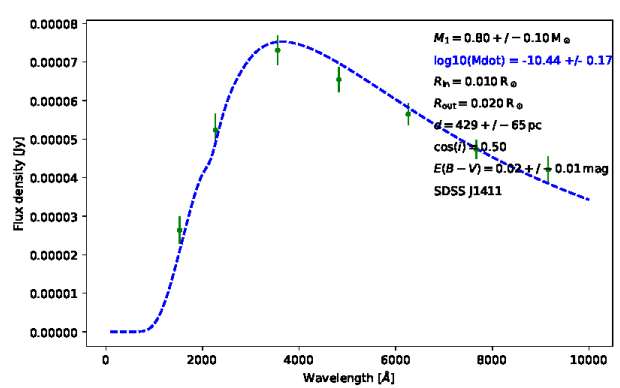

(a)

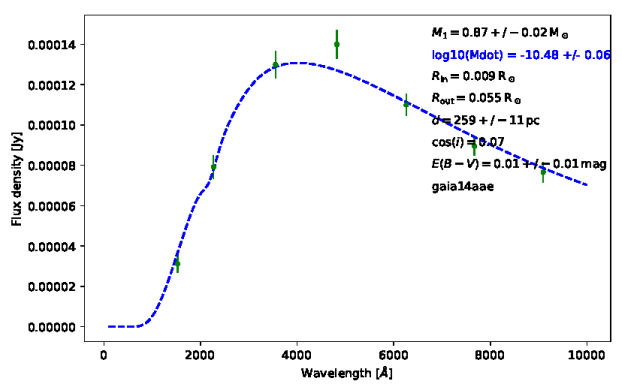

(c)

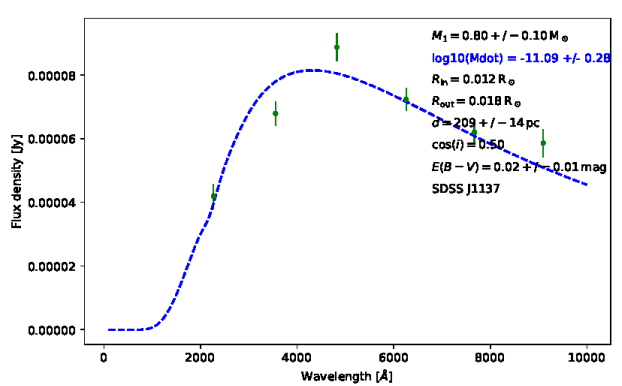

(e)

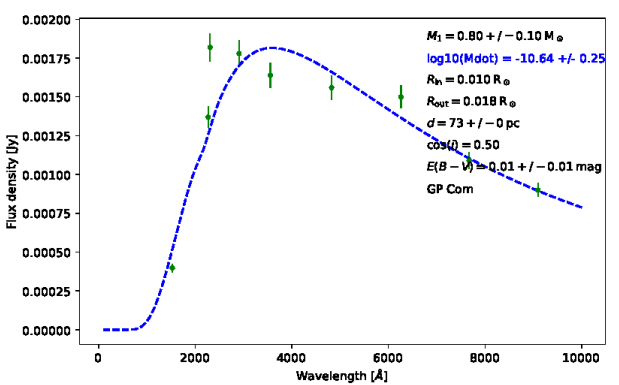

(b)

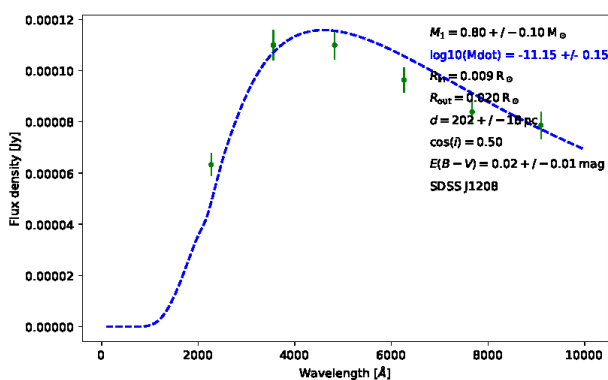

(d)

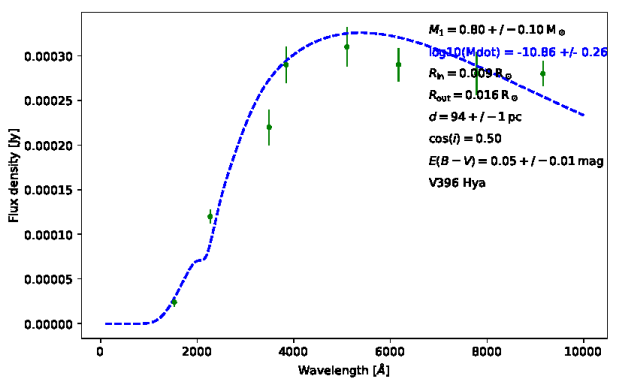

(f)

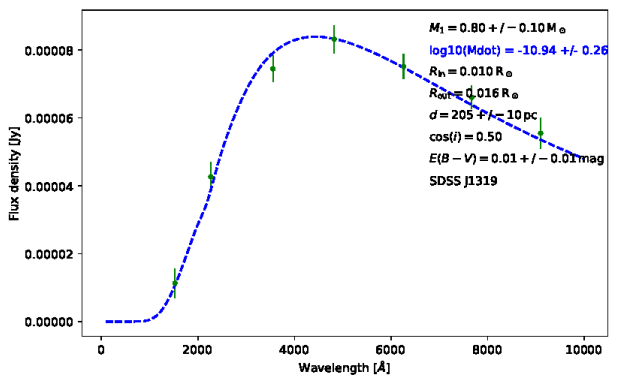

(g)

Fig. B.1. continued. 
Table B.1. Kinematic information for the AM CVn stars with measured proper motions in Gaia DR2.

\begin{tabular}{|c|c|c|c|}
\hline Source & $\begin{array}{r}\text { pmra } \\
\left(\mathrm{mas} \mathrm{yr}^{-1}\right) \\
\end{array}$ & $\begin{array}{r}\text { pmdec } \\
\left(\mathrm{mas} \mathrm{yr}^{-1}\right)\end{array}$ & Population \\
\hline V407 Vul & $-2.315 \pm 0.386$ & $-3.726 \pm 0.719$ & Thin \\
\hline ES Cet & $17.486 \pm 0.196$ & $-3.075 \pm 0.157$ & Thick \\
\hline SDSS J1351-0643 & $5.503 \pm 0.586$ & $-17.328 \pm 0.465$ & Thick \\
\hline AM CVn & $30.935 \pm 0.073$ & $12.420 \pm 0.053$ & Thick \\
\hline SDSS J1908+3940 & $-7.019 \pm 0.087$ & $-5.332 \pm 0.093$ & Thin \\
\hline HP Lib & $-28.776 \pm 0.099$ & $-12.636 \pm 0.074$ & Thin \\
\hline PTF1 J1919+4815 & $5.548 \pm 0.535$ & $5.850 \pm 0.830$ & Thin/thick \\
\hline CX361 & $2.393 \pm 0.344$ & $-2.001 \pm 0.288$ & Thin \\
\hline ASASSN-14cc & $-14.897 \pm 0.115$ & $-37.800 \pm 0.111$ & Thick \\
\hline KL Dra & $-2.463 \pm 0.296$ & $-18.269 \pm 0.262$ & Thick \\
\hline PTF1 J0719+4858 & $4.276 \pm 0.297$ & $2.345 \pm 0.166$ & Thin \\
\hline YZ LMi & $-30.506 \pm 0.841$ & $-4.072 \pm 1.084$ & Thin \\
\hline CP Eri & $18.090 \pm 1.445$ & $-7.174 \pm 1.550$ & Thin \\
\hline SDSS J1043+5632 & $-0.561 \pm 0.936$ & $-2.997 \pm 1.099$ & Thin \\
\hline CRTS J0910-2008 & $3.730 \pm 1.050$ & $-8.610 \pm 0.923$ & Thin/thick \\
\hline CRTS J0105+1903 & $7.159 \pm 1.023$ & $8.640 \pm 0.923$ & Thin \\
\hline V406 Нyа & $-1.429 \pm 1.606$ & $-6.191 \pm 1.389$ & Thin \\
\hline SDSS J1730+5545 & $4.104 \pm 0.829$ & $-33.861 \pm 0.931$ & Thick \\
\hline NSV1440 & $7.737 \pm 0.255$ & $27.446 \pm 0.277$ & Thin \\
\hline V558 Vir & $-13.989 \pm 2.482$ & $-3.959 \pm 3.140$ & Thin \\
\hline SDSS J1240-0159 & $-1.852 \pm 1.248$ & $-8.006 \pm 0.646$ & Thin \\
\hline V744 And & $-2.691 \pm 0.827$ & $6.332 \pm 1.797$ & Thick \\
\hline SDSS J1721+2733 & $2.815 \pm 0.923$ & $-2.025 \pm 1.102$ & Thin \\
\hline ASASSN-14mv & $-17.270 \pm 0.266$ & $-43.836 \pm 0.205$ & Thin \\
\hline ASASSN-14ei & $-10.263 \pm 0.074$ & $-15.380 \pm 0.098$ & Thin \\
\hline SDSS J1525+3600 & $4.787 \pm 0.518$ & $-16.876 \pm 0.810$ & Thin \\
\hline SDSS J0804+1616 & $1.365 \pm 0.382$ & $-5.972 \pm 0.219$ & Thin \\
\hline SDSS J1411+4812 & $-17.297 \pm 0.431$ & $36.323 \pm 0.444$ & Thick \\
\hline GP Com & $-344.791 \pm 0.131$ & $34.743 \pm 0.082$ & Thin/thick \\
\hline SDSS J0902+3819 & $-15.799 \pm 1.170$ & $-13.360 \pm 1.058$ & Thin \\
\hline Gaia14aae & $-3.927 \pm 0.317$ & $-14.099 \pm 0.269$ & Thin \\
\hline SDSS J1208+3550 & $-112.865 \pm 0.651$ & $-56.236 \pm 0.510$ & Thick \\
\hline SDSS J1642+1934 & $-2.560 \pm 1.034$ & $-28.606 \pm 1.082$ & Thick \\
\hline SDSS J1552+3201 & $5.943 \pm 0.947$ & $-27.253 \pm 1.174$ & Thin \\
\hline SDSS J1137+4054 & $23.118 \pm 0.383$ & $-56.566 \pm 0.336$ & Thin \\
\hline V396 Нуа & $-286.331 \pm 0.306$ & $-59.209 \pm 0.213$ & Thick \\
\hline SDSS J1319+5915 & $-27.885 \pm 0.498$ & $12.375 \pm 0.359$ & Thin \\
\hline CRTS J0844-0128 & $-5.291 \pm 1.621$ & $-4.794 \pm 0.549$ & Thin \\
\hline SDSS J1505+0659 & $44.487 \pm 0.724$ & $-25.417 \pm 0.686$ & Thin/thick \\
\hline Gaia 16all & $-2.640 \pm 1.647$ & $5.287 \pm 1.465$ & Thin \\
\hline
\end{tabular}

Notes. These data are also included in the online table. 